\title{
Hierarchical Self-Assembly of Aminopyrazole Peptides into Nanorosettes in Water
}

Petra Rzepecki, Katrin Hochdörffer, Torsten Schaller, Jan Zienau, Klaus Harms, Christian Ochsenfeld*, Xiulan Xie*, Thomas Schrader*

\section{Supporting Information}

\section{Table of Contents:}

1. X-ray crystallography: structure models

Tables S1-S8: crystal data

2. NMR spectroscopy: ${ }^{1} \mathrm{H}$ NMR spectrum at $298 \mathrm{~K}$

${ }^{13} \mathrm{C}$ NMR spectrum at $298 \mathrm{~K}$

Variable temperature ${ }^{1} \mathrm{H}$ NMR experiment

${ }^{1} \mathrm{H}$ NMR spectrum at $275 \mathrm{~K}$

3. Quantum chemical calculations: NMR shift tables 


\section{X-ray crystallography:}

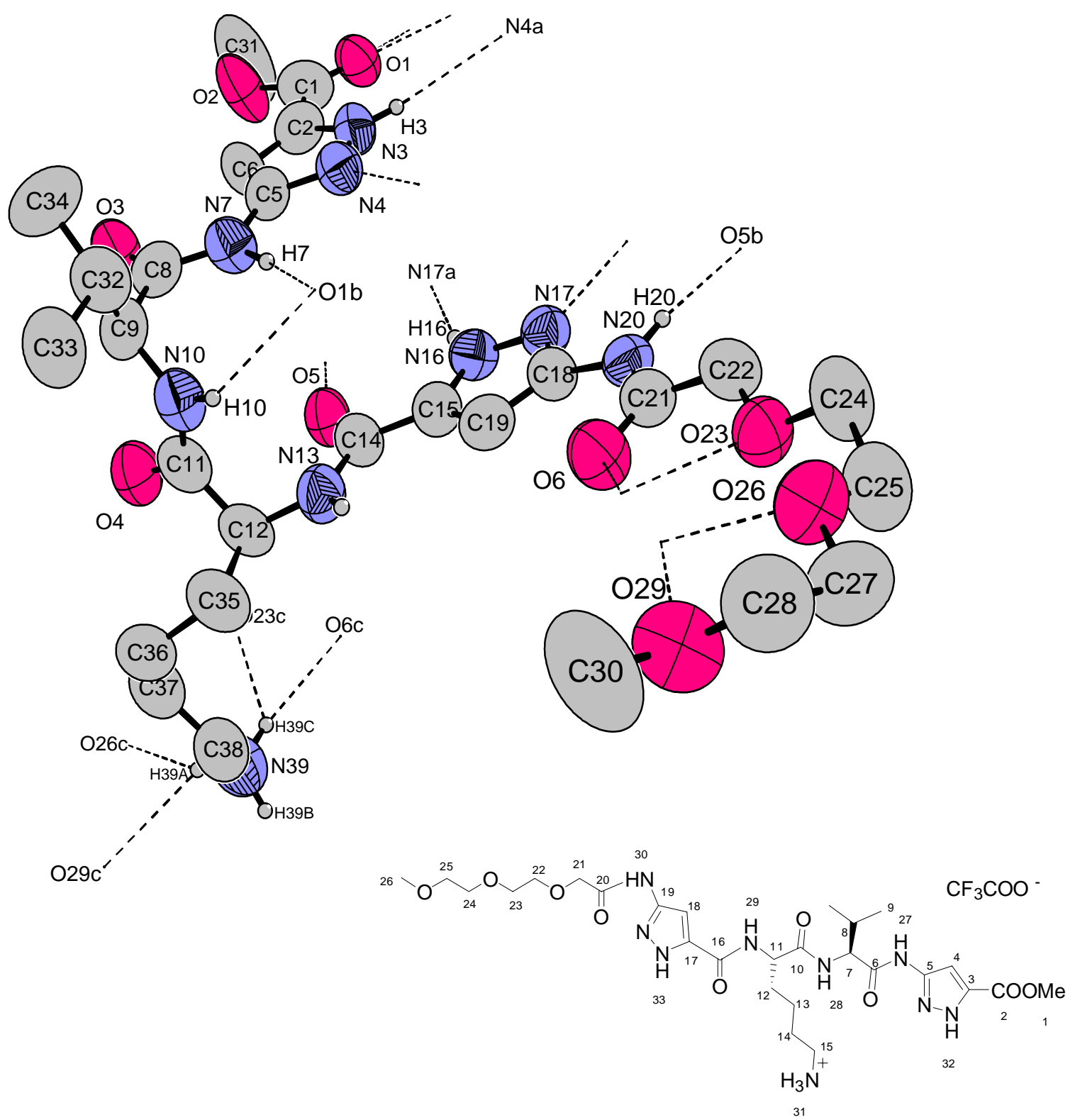

Figure S1. Monomer with hydrogen bond contacts, presented in perspectivic depth view. (carbon: black; hydrogen: white; oxygen: red, nitrogen: blue). Hydrogen bonds are dotted black lines. 


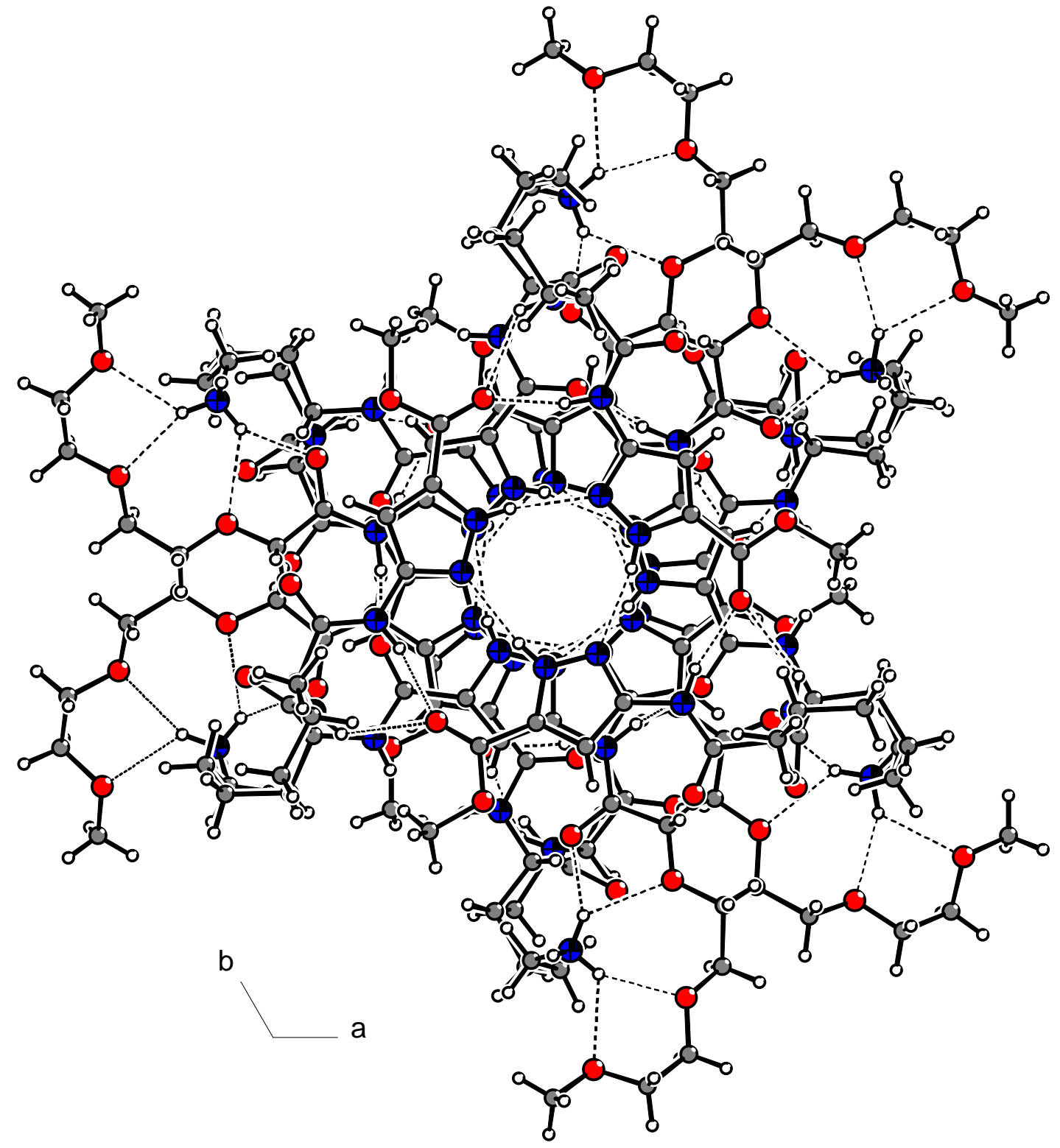

Figure S2. Hexameric aggregate: top view (carbon: black; hydrogen: white; oxygen: red, nitrogen: blue). Hydrogen bonds are dotted black lines. 


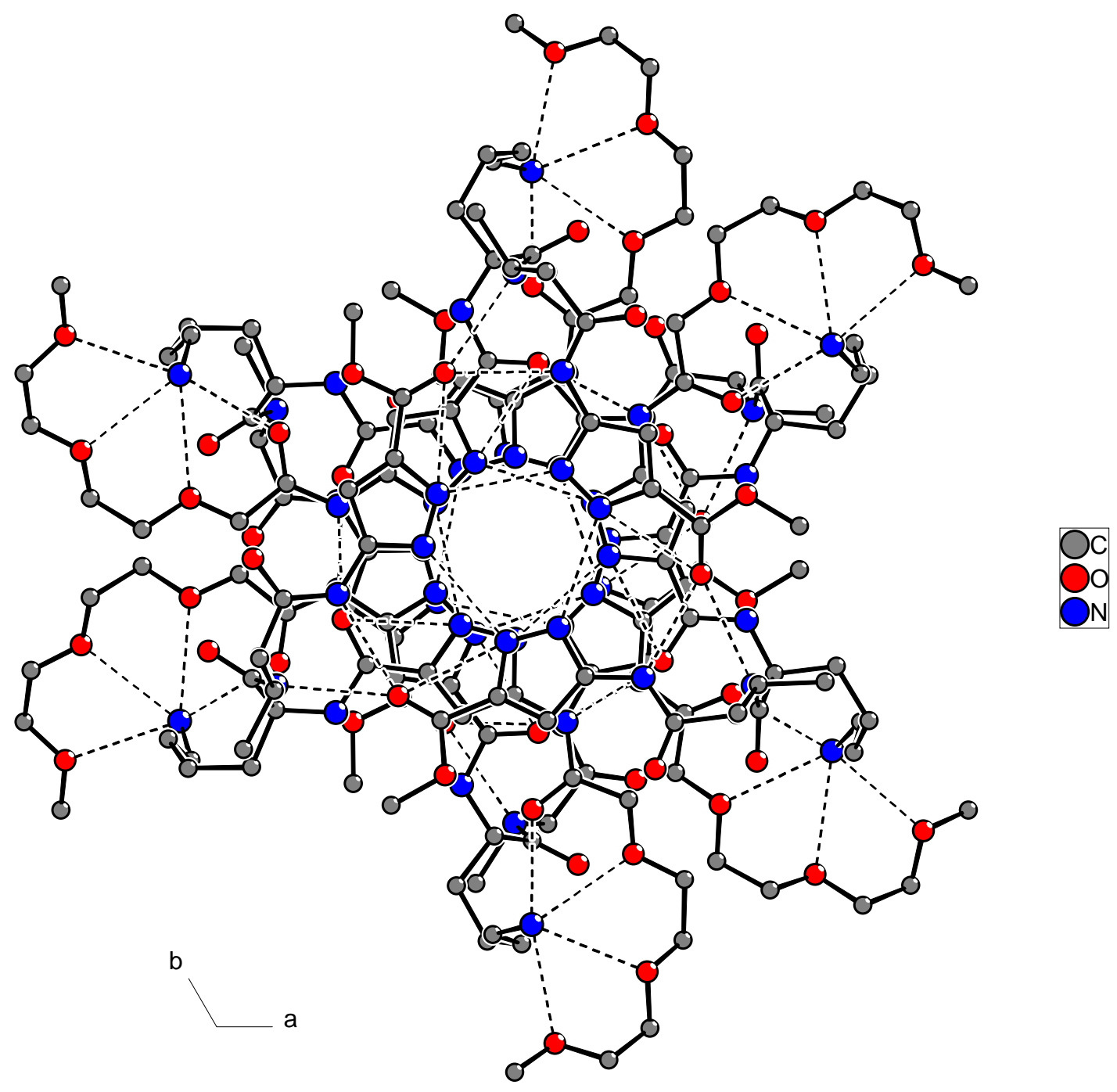

Figure S3. Hexameric aggregate: top view (carbon: black; hydrogen atoms omitted for clarity; oxygen: red, nitrogen: blue). Hydrogen bonds are dotted black lines. 


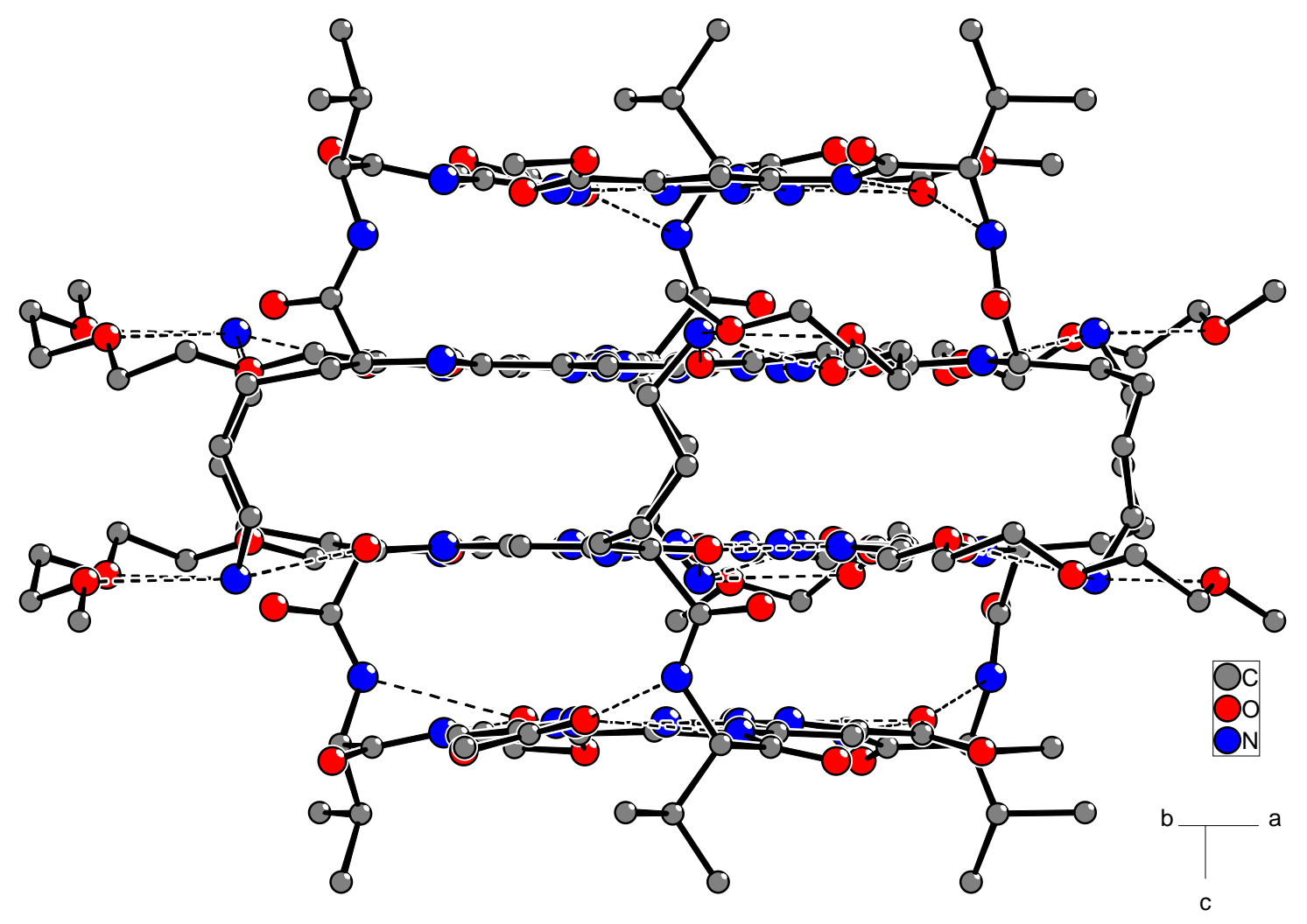

Figure S4. Hexameric aggregate: side view (carbon: black; hydrogen atoms omitted for clarity; oxygen: red, nitrogen: blue). Hydrogen bonds are dotted black lines. 


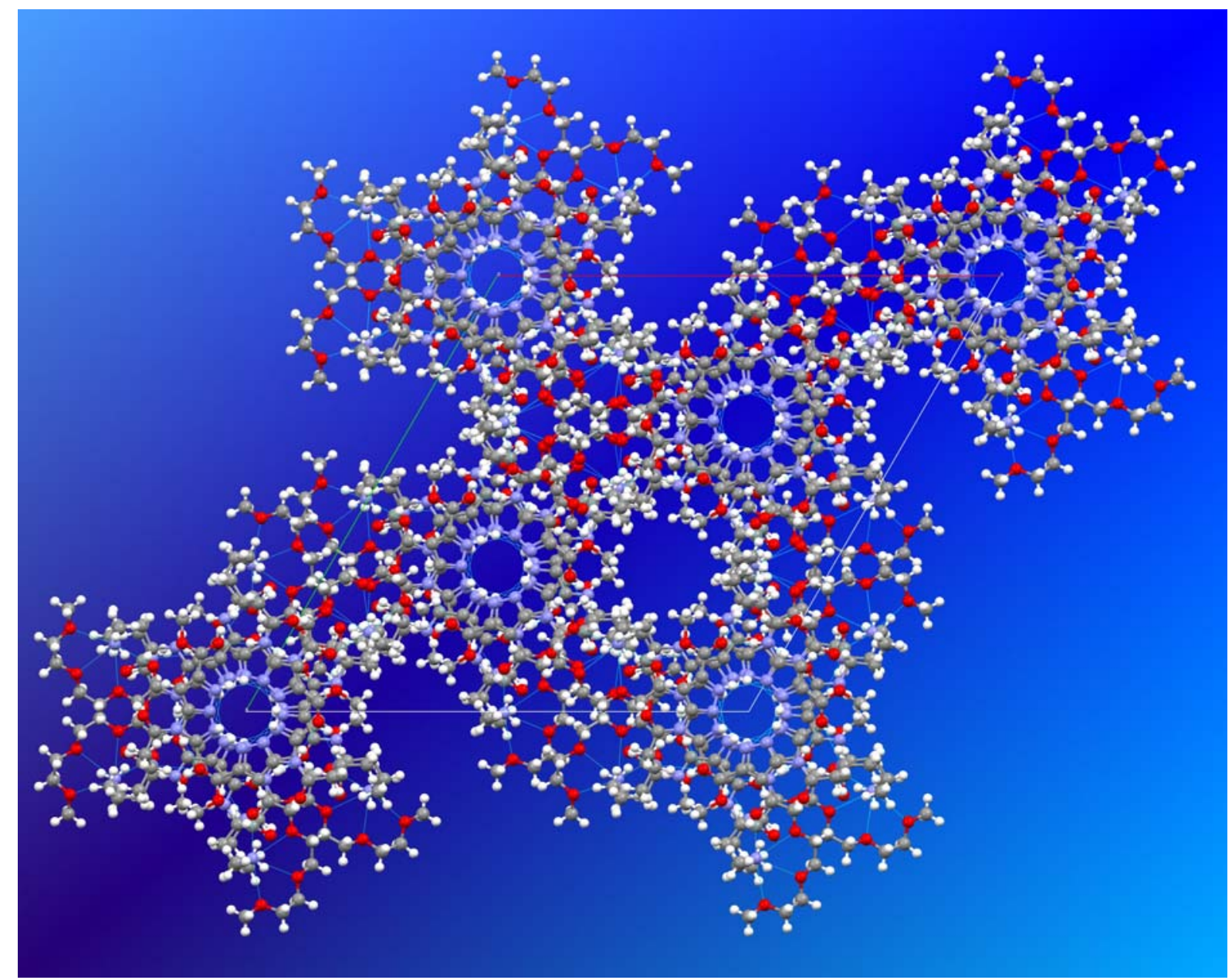

Figure S 5. Crystal packing of nanorosettes in the unit cell (ball and stick representation; carbon: black; hydrogen: white; oxygen: red, nitrogen: blue). Hydrogen bonds are dotted light blue lines. Note the central cavity, formed in the crystal: only nonpolar groups are pointing into its interior, minimizing attractive forces onto the trifluoroacetate counterions, which remain heavily disordered. 


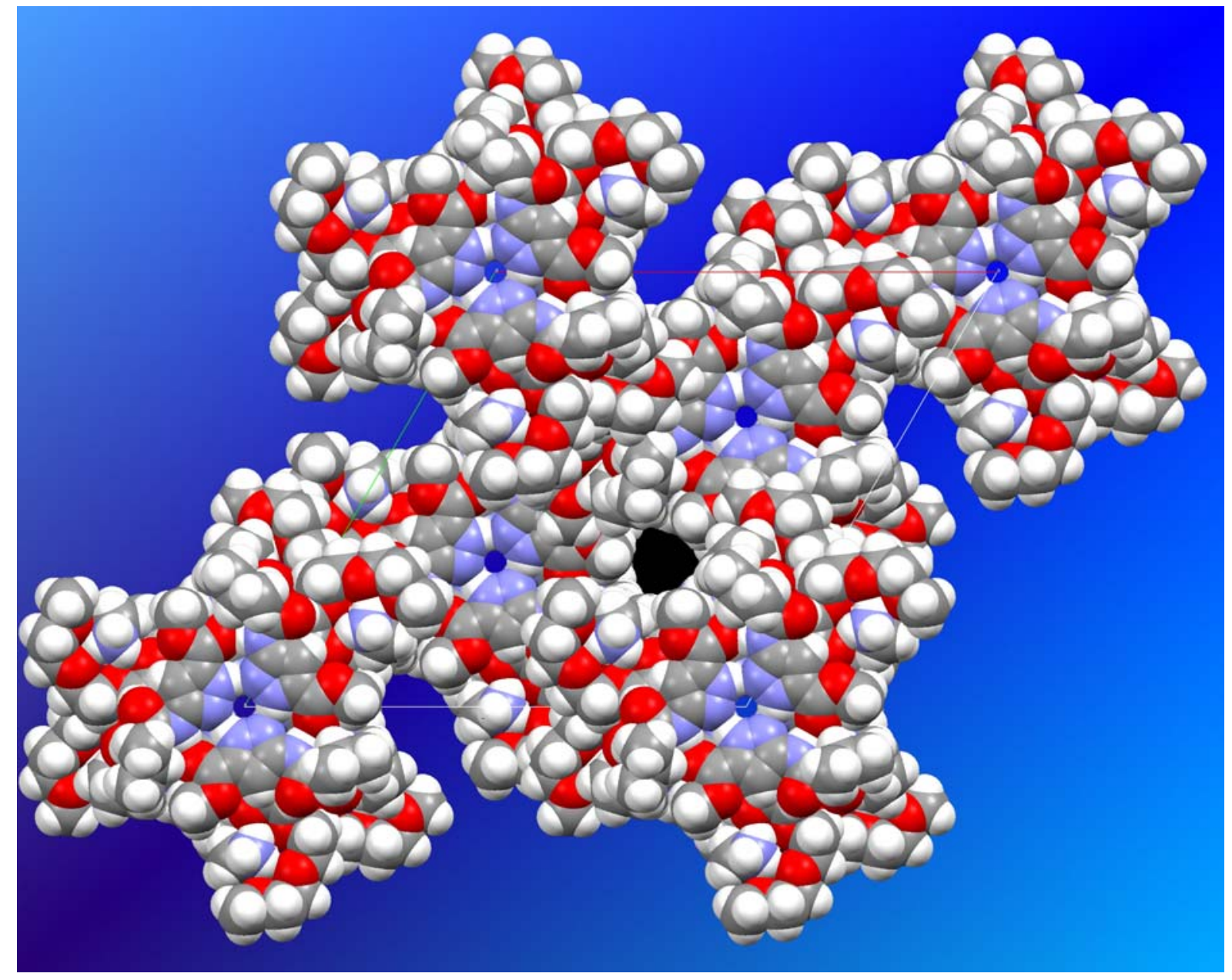

Figure S6. Crystal packing of nanorosettes in the unit cell (space filling representation; carbon: black; hydrogen: white; oxygen: red, nitrogen: blue). Hydrogen bonds are dotted light blue lines. Note the central cavity in black, formed in the crystal: only nonpolar groups are pointing into its interior, minimizing attractive forces onto the trifluoroacetate counterions, which remain heavily disordered. 
Table S1. Crystal data and structure refinement for aminopyrazole hybrid peptide $\mathbf{1}$.

Crystal data

Identification code

Habitus, colour

Crystal size

Crystal system

Space group

Unit cell dimensions

Volume

Cell determination

Empirical formula

Formula weight

Density (calculated)

Absorption coefficient

$\mathrm{F}(000)$

Data collection:

Diffractometer type

Wavelength

Temperature

Theta range for data collection

Index ranges

Data collection software

Cell refinement software

Data reduction software

Solution and refinement:

Reflections collected

Independent reflections

Completeness to theta $=24.99^{\circ}$

Observed reflections

Reflections used for refinement

Absorption correction

Flack parameter (absolute struct.)

Largest diff. peak and hole

Solution

Refinement

Treatment of hydrogen atoms

Programs used

Data / restraints / parameters

Goodness-of-fit on $\mathrm{F}^{2}$

$\mathrm{R}$ index (all data)

$\mathrm{R}$ index conventional [I $>2 \operatorname{sigma}(\mathrm{I})]$
63567

4pet45

cubic, colourless

$0.35 \times 0.33 \times 0.33 \mathrm{~mm}^{3}$

Trigonal

R 32

$\mathrm{Z}=18$

$\mathrm{a}=30.9179(10) \AA$

$\alpha=90^{\circ}$.

$\mathrm{b}=30.9179(10) \AA$

$\beta=90^{\circ}$.

$\mathrm{c}=35.2839(15) \AA$

29209.7(18) $\AA^{3}$

$\gamma=120^{\circ}$

56521 peaks with Theta 1.5 to $24.5^{\circ}$.

$\mathrm{C}_{41} \mathrm{H}_{74} \mathrm{~F}_{3} \mathrm{~N}_{9} \mathrm{O}_{14}$

$\mathrm{C}_{27} \mathrm{H}_{44} \mathrm{~N}_{9} \mathrm{O}_{9}, \mathrm{C} \mathrm{F}_{3} \mathrm{C} \mathrm{O}_{2}, 3\left(\mathrm{C}_{4} \mathrm{H}_{10} \mathrm{O}\right)$

974.09

$0.997 \mathrm{Mg} / \mathrm{m}^{3}$

$0.080 \mathrm{~mm}^{-1}$

9396

IPDS2

$0.71073 \AA$

123(2) K

1.32 to $24.99^{\circ}$.

$-36<=\mathrm{h}<=36,-35<=\mathrm{k}<=36,-41<=\mathrm{l}<=41$

STOE WinXpose (X-Area)

STOE WinCell (X-Area)

STOE WinIntegrate (X-Area)

$11252[\mathrm{R}(\mathrm{int})=0.0724]$

$98.5 \%$

$5650[\mathrm{I}>2 \operatorname{sigma}(\mathrm{I})]$

11252

None

$0.3(9)$

0.107 and -0.121 e. $\AA^{-3}$

Direct methods

Full-matrix least-squares on $\mathrm{F}^{2}$

Calculated positions, constr. ref.

SHELXD (Sheldrick, 2002)

SHELXL-97 (Sheldrick, 1997)

Diamond 3.1, STOE IPDS2 software

11252 / 0 / 411

0.752

$\mathrm{wR} 2=0.1001$

$\mathrm{R} 1=0.0434$ 
Table S2. Atomic coordinates and equivalent isotropic displacement parameters $\left(\AA^{2}\right)$ for aminopyrazole hybrid peptide 1. $\mathrm{U}(\mathrm{eq})$ is defined as one third of the trace of the orthogonalized $\mathrm{U}^{\mathrm{ij}}$ tensor.

\begin{tabular}{|c|c|c|c|c|c|}
\hline & $\mathrm{x}$ & $\mathrm{y}$ & $\mathrm{z}$ & $\mathrm{U}(\mathrm{eq})$ & Occupancy \\
\hline $\mathrm{C} 1$ & $0.97887(13)$ & $0.13402(11)$ & $0.14389(8)$ & $0.0969(8)$ & 1 \\
\hline $\mathrm{C} 2$ & $0.94699(10)$ & $0.08005(9)$ & $0.14223(6)$ & $0.0811(6)$ & 1 \\
\hline $\mathrm{C} 5$ & $0.88823(10)$ & $0.00395(10)$ & $0.14336(6)$ & $0.0785(7)$ & 1 \\
\hline C6 & $0.89712(9)$ & $0.05217(10)$ & $0.14469(7)$ & $0.0812(7)$ & 1 \\
\hline $\mathrm{C} 8$ & $0.79680(10)$ & $-0.04550(11)$ & $0.15063(7)$ & $0.0827(7)$ & 1 \\
\hline C9 & 0.75231(9) & $-0.09769(9)$ & $0.14935(7)$ & $0.0836(7)$ & 1 \\
\hline C11 & $0.73746(9)$ & $-0.11720(10)$ & $0.08176(8)$ & $0.0877(7)$ & 1 \\
\hline C12 & $0.74145(8)$ & $-0.14505(8)$ & $0.04797(8)$ & $0.0848(7)$ & 1 \\
\hline C14 & $0.83301(9)$ & $-0.10488(11)$ & $0.04817(7)$ & $0.0782(7)$ & 1 \\
\hline C15 & $0.87675(9)$ & $-0.11155(9)$ & $0.04722(7)$ & $0.0765(6)$ & 1 \\
\hline C18 & $0.93267(10)$ & $-0.13214(10)$ & $0.04638(6)$ & $0.0790(6)$ & 1 \\
\hline C19 & $0.88318(9)$ & $-0.15138(9)$ & $0.04728(6)$ & $0.0742(6)$ & 1 \\
\hline C21 & $0.94209(11)$ & $-0.20624(10)$ & $0.04854(7)$ & $0.0833(7)$ & 1 \\
\hline C22 & $0.97643(9)$ & $-0.22559(10)$ & $0.05322(7)$ & $0.0910(7)$ & 1 \\
\hline C24 & $0.98415(10)$ & $-0.29753(10)$ & $0.05440(9)$ & $0.1093(9)$ & 1 \\
\hline C25 & $0.95606(12)$ & $-0.35105(11)$ & $0.03966(10)$ & $0.1194(10)$ & 1 \\
\hline $\mathrm{C} 27$ & $0.88933(12)$ & $-0.43142(10)$ & $0.05079(9)$ & $0.1137(9)$ & 1 \\
\hline C28 & $0.84339(12)$ & $-0.46001(11)$ & $0.07583(9)$ & $0.1131(9)$ & 1 \\
\hline C30 & $0.76514(14)$ & $-0.46980(14)$ & $0.08570(12)$ & $0.1678(15)$ & 1 \\
\hline C31 & $0.98051(12)$ & $0.21143(11)$ & $0.14957(14)$ & $0.188(2)$ & 1 \\
\hline C32 & 0.74837(9) & $-0.12805(9)$ & $0.18552(7)$ & $0.0947(7)$ & 1 \\
\hline C33 & $0.69793(11)$ & $-0.18046(10)$ & $0.18361(9)$ & $0.1296(11)$ & 1 \\
\hline C34 & $0.75164(12)$ & $-0.09928(10)$ & $0.22091(7)$ & $0.1146(9)$ & 1 \\
\hline C35 & $0.69614(9)$ & $-0.19703(10)$ & $0.04557(8)$ & $0.0974(8)$ & 1 \\
\hline C36 & $0.64705(9)$ & $-0.19789(10)$ & $0.03788(8)$ & $0.0989(8)$ & 1 \\
\hline C37 & $0.64453(9)$ & $-0.17183(11)$ & $0.00459(7)$ & $0.0979(8)$ & 1 \\
\hline C38 & $0.65329(10)$ & $-0.19088(10)$ & $-0.03193(8)$ & $0.0998(8)$ & 1 \\
\hline N3 & $0.96515(7)$ & $0.04941(8)$ & $0.13795(5)$ & $0.0791(5)$ & 1 \\
\hline N4 & $0.92952(7)$ & $0.00120(7)$ & $0.13834(5)$ & $0.0739(5)$ & 1 \\
\hline N7 & $0.84203(8)$ & $-0.04121(7)$ & $0.14377(5)$ & $0.0825(5)$ & 1 \\
\hline N10 & $0.75285(6)$ & $-0.12351(8)$ & $0.11420(7)$ & $0.0877(5)$ & 1 \\
\hline N13 & $0.78698(7)$ & $-0.14784(8)$ & $0.04945(5)$ & $0.0862(6)$ & 1 \\
\hline N16 & $0.92295(7)$ & $-0.06984(7)$ & $0.04692(5)$ & $0.0717(5)$ & 1 \\
\hline N17 & $0.95940(7)$ & $-0.08177(7)$ & $0.04607(5)$ & $0.0724(5)$ & 1 \\
\hline N20 & $0.96237(8)$ & $-0.15588(8)$ & $0.04679(5)$ & $0.0815(6)$ & 1 \\
\hline N39 & $0.66276(8)$ & $-0.15509(8)$ & $-0.06337(6)$ & $0.0982(6)$ & 1 \\
\hline $\mathrm{O} 1$ & 1.02399(7) & $0.15673(6)$ & $0.13646(5)$ & $0.0885(5)$ & 1 \\
\hline $\mathrm{O} 2$ & $0.95211(6)$ & $0.15625(6)$ & $0.15208(7)$ & $0.1204(7)$ & 1 \\
\hline O3 & $0.79192(6)$ & $-0.00947(7)$ & $0.15777(5)$ & $0.0921(5)$ & 1 \\
\hline $\mathrm{O} 4$ & $0.71620(7)$ & $-0.09227(7)$ & $0.07835(5)$ & $0.1046(6)$ & 1 \\
\hline O5 & $0.83485(6)$ & $-0.06399(7)$ & $0.04835(5)$ & $0.0862(5)$ & 1 \\
\hline O6 & $0.89627(7)$ & $-0.23528(7)$ & $0.04755(5)$ & $0.1007(5)$ & 1 \\
\hline $\mathrm{O} 23$ & $0.95473(6)$ & $-0.27518(6)$ & $0.04407(5)$ & $0.0951(5)$ & 1 \\
\hline $\mathrm{O} 26$ & $0.91333(7)$ & $-0.37980(7)$ & $0.06164(5)$ & $0.0985(5)$ & 1 \\
\hline O29 & $0.80951(8)$ & $-0.44382(7)$ & $0.06477(5)$ & $0.1110(6)$ & 1 \\
\hline
\end{tabular}


Table S3. Bond lengths $[\AA]$ and angles $\left[{ }^{\circ}\right]$ for aminopyrazole hybrid peptide $\mathbf{1}$.

\begin{tabular}{|c|c|c|c|}
\hline$\overline{\mathrm{C} 1-\mathrm{O} 1}$ & $1.236(3)$ & C27-H27A & 0.9900 \\
\hline $\mathrm{C} 1-\mathrm{O} 2$ & $1.345(3)$ & C27-H27B & 0.9900 \\
\hline $\mathrm{C} 1-\mathrm{C} 2$ & $1.454(3)$ & C28-029 & $1.423(3)$ \\
\hline C2-N3 & $1.329(3)$ & C28-H28A & 0.9900 \\
\hline C2-C6 & 1.341(3) & C28-H28B & 0.9900 \\
\hline C5-N4 & 1.333(3) & C30-O29 & $1.404(4)$ \\
\hline C5-C6 & $1.375(3)$ & C30-H30A & 0.9800 \\
\hline C5-N7 & $1.413(3)$ & С30-Н30В & 0.9800 \\
\hline C6-H6 & 0.9500 & С30-Н30С & 0.9800 \\
\hline C8-O3 & $1.223(3)$ & C31-O2 & $1.480(3)$ \\
\hline C8-N7 & $1.359(3)$ & C31-H31A & 0.9800 \\
\hline C8-C9 & $1.510(3)$ & С31-Н31B & 0.9800 \\
\hline C9-N10 & $1.480(3)$ & С31-Н31C & 0.9800 \\
\hline C9-C32 & $1.552(3)$ & C32-C34 & $1.507(3)$ \\
\hline C9-H9 & 1.0000 & C32-C33 & $1.592(3)$ \\
\hline C11-O4 & $1.244(3)$ & C32-H32 & 1.0000 \\
\hline C11-N10 & 1.291(3) & С33-Н33А & 0.9800 \\
\hline C11-C12 & 1.512(3) & С33-Н33В & 0.9800 \\
\hline C12-N13 & $1.454(3)$ & С33-Н33С & 0.9800 \\
\hline C12-C35 & $1.517(3)$ & С34-H34A & 0.9800 \\
\hline C12-H12 & 1.0000 & С34-Н34B & 0.9800 \\
\hline C14-O5 & $1.237(3)$ & С34-Н34C & 0.9800 \\
\hline C14-N13 & $1.379(3)$ & C35-C36 & $1.529(3)$ \\
\hline C14-C15 & $1.467(3)$ & С35-Н35A & 0.9900 \\
\hline C15-C19 & $1.342(3)$ & С35-Н35B & 0.9900 \\
\hline C15-N16 & $1.365(3)$ & C36-C37 & $1.448(3)$ \\
\hline C18-C19 & 1.336(3) & C36-H36A & 0.9900 \\
\hline C18-N17 & $1.349(3)$ & С36-H36B & 0.9900 \\
\hline C18-N20 & $1.434(3)$ & C37-C38 & $1.497(3)$ \\
\hline C19-H19 & 0.9500 & С37-H37A & 0.9900 \\
\hline C21-O6 & $1.242(3)$ & C37-H37B & 0.9900 \\
\hline C21-N20 & $1.358(3)$ & C38-N39 & $1.489(3)$ \\
\hline C21-C22 & $1.466(3)$ & C38-H38A & 0.9900 \\
\hline C22-O23 & $1.370(3)$ & С38-Н38B & 0.9900 \\
\hline C22-H22A & 0.9900 & N3-N4 & $1.339(2)$ \\
\hline C22-H22B & 0.9900 & N3-H3 & 0.8800 \\
\hline $\mathrm{C} 24-\mathrm{O} 23$ & $1.437(3)$ & N7-H7 & 0.8800 \\
\hline C24-C25 & $1.525(4)$ & N10-H10 & 0.8800 \\
\hline C24-H24A & 0.9900 & N13-H13 & 0.8800 \\
\hline C24-H24B & 0.9900 & N16-N17 & $1.350(2)$ \\
\hline C25-O26 & $1.401(3)$ & N16-H16 & 0.8800 \\
\hline C25-H25A & 0.9900 & N20-H20 & 0.8800 \\
\hline C25-H25B & 0.9900 & N39-H39A & 0.9100 \\
\hline C27-O26 & $1.435(3)$ & N39-H39B & 0.9100 \\
\hline C27-C28 & $1.524(4)$ & N39-H39C & 0.9100 \\
\hline O1-C1-O2 & $124.3(2)$ & N7-C8-C9 & $116.2(2)$ \\
\hline O1-C1-C2 & 124.6(3) & N10-C9-C8 & 110.61(19) \\
\hline O2-C1-C2 & 111.0(3) & N10-C9-C32 & $112.4(2)$ \\
\hline N3-C2-C6 & $108.0(2)$ & C8-C9-C32 & $112.1(2)$ \\
\hline N3-C2-C1 & 122.5(3) & N10-C9-H9 & 107.1 \\
\hline C6-C2-C1 & $129.5(2)$ & С8-C9-H9 & 107.1 \\
\hline N4-C5-C6 & $113.3(2)$ & С32-С9-H9 & 107.1 \\
\hline N4-C5-N7 & $117.8(2)$ & O4-C11-N10 & 121.9(3) \\
\hline C6-C5-N7 & $128.8(2)$ & O4-C11-C12 & 119.7(3) \\
\hline C2-C6-C5 & 103.7(2) & N10-C11-C12 & 118.2(3) \\
\hline C2-C6-H6 & 128.1 & N13-C12-C11 & $111.3(2)$ \\
\hline C5-C6-H6 & 128.1 & N13-C12-C35 & $110.3(2)$ \\
\hline O3-C8-N7 & 122.5(3) & C11-C12-C35 & $110.6(2)$ \\
\hline O3-C8-C9 & $121.2(2)$ & N13-C12-H12 & 108.2 \\
\hline
\end{tabular}




\begin{tabular}{|c|c|}
\hline C11-C12-H12 & 108.2 \\
\hline C35-C12-H12 & 108.2 \\
\hline O5-C14-N13 & $118.8(2)$ \\
\hline O5-C14-C15 & $124.7(2)$ \\
\hline N13-C14-C15 & $116.4(2)$ \\
\hline C19-C15-N16 & $107.6(2)$ \\
\hline C19-C15-C14 & $134.4(2)$ \\
\hline N16-C15-C14 & 118.1(2) \\
\hline C19-C18-N17 & $114.7(2)$ \\
\hline C19-C18-N20 & $131.0(2)$ \\
\hline N17-C18-N20 & $114.3(2)$ \\
\hline C18-C19-C15 & $104.7(2)$ \\
\hline C18-C19-H19 & 127.7 \\
\hline C15-C19-H19 & 127.7 \\
\hline O6-C21-N20 & $122.2(2)$ \\
\hline O6-C21-C22 & $120.3(2)$ \\
\hline N20-C21-C22 & $117.4(2)$ \\
\hline O23-C22-C21 & $112.4(2)$ \\
\hline O23-C22-H22A & 109.1 \\
\hline C21-C22-H22A & 109.1 \\
\hline O23-C22-H22B & 109.1 \\
\hline C21-C22-H22B & 109.1 \\
\hline H22A-C22-H22B & 107.9 \\
\hline O23-C24-C25 & $106.3(2)$ \\
\hline O23-C24-H24A & 110.5 \\
\hline C25-C24-H24A & 110.5 \\
\hline O23-C24-H24B & 110.5 \\
\hline C25-C24-H24B & 110.5 \\
\hline H24A-C24-H24B & 108.7 \\
\hline O26-C25-C24 & $110.1(2)$ \\
\hline O26-C25-H25A & 109.6 \\
\hline C24-C25-H25A & 109.6 \\
\hline O26-C25-H25B & 109.6 \\
\hline C24-C25-H25B & 109.6 \\
\hline H25A-C25-H25B & 108.1 \\
\hline O26-C27-C28 & $107.7(2)$ \\
\hline O26-C27-H27A & 110.2 \\
\hline C28-C27-H27A & 110.2 \\
\hline O26-C27-H27B & 110.2 \\
\hline C28-C27-H27B & 110.2 \\
\hline H27A-C27-H27B & 108.5 \\
\hline O29-C28-C27 & $105.9(2)$ \\
\hline O29-C28-H28A & 110.6 \\
\hline C27-C28-H28A & 110.6 \\
\hline O29-С28-H28B & 110.6 \\
\hline C27-C28-H28B & 110.6 \\
\hline H28A-C28-H28B & 108.7 \\
\hline O29-С30-H30A & 109.5 \\
\hline О29-С30-Н30В & 109.5 \\
\hline Н30А-СЗ0-Н30В & 109.5 \\
\hline О29-С30-Н30С & 109.5 \\
\hline Н30А-С30-Н30С & 109.5 \\
\hline Н30В-С30-Н30С & 109.5 \\
\hline O2-C31-H31A & 109.5 \\
\hline O2-C31-Н31B & 109.5 \\
\hline Н31А-С31-Н31B & 109.5 \\
\hline O2-C31-H31C & 109.5 \\
\hline H31А-С31-Н31C & 109.5 \\
\hline Н31B-С31-Н31C & 109.5 \\
\hline С34-C32-C9 & $111.3(2)$ \\
\hline C34-C32-C33 & $112.2(2)$ \\
\hline C9-C32-C33 & $108.7(2)$ \\
\hline
\end{tabular}

\begin{tabular}{|c|c|}
\hline С34-С32-H32 & 108.2 \\
\hline С9-С32-Н32 & 108.2 \\
\hline С33-С32-Н32 & 108.2 \\
\hline С32-С33-Н33А & 109.5 \\
\hline С32-С33-НЗ3В & 109.5 \\
\hline НЗЗА-СЗ3-НЗЗВ & 109.5 \\
\hline С32-С33-Н33С & 109.5 \\
\hline Н3ЗА-СЗ3-Н33С & 109.5 \\
\hline НЗ3В-СЗ3-Н33С & 109.5 \\
\hline C32-C34-H34A & 109.5 \\
\hline С32-С34-Н34В & 109.5 \\
\hline Н34А-С34-Н34В & 109.5 \\
\hline С32-С34-H34C & 109.5 \\
\hline Н34А-С34-Н34С & 109.5 \\
\hline Н34В-С34-Н34С & 109.5 \\
\hline C12-C35-C36 & $114.3(2)$ \\
\hline C12-С35-Н35A & 108.7 \\
\hline С36-С35-H35A & 108.7 \\
\hline С12-С35-Н35В & 108.7 \\
\hline С36-С35-Н35В & 108.7 \\
\hline Н35А-С35-Н35В & 107.6 \\
\hline C37-C36-C35 & $117.6(2)$ \\
\hline С37-С36-Н36А & 107.9 \\
\hline С35-С36-Н36А & 107.9 \\
\hline С37-С36-Н36В & 107.9 \\
\hline С35-С36-Н36В & 107.9 \\
\hline Н36А-С36-Н36В & 107.2 \\
\hline C36-С37-C38 & 114.1(3) \\
\hline С36-С37-Н37А & 108.7 \\
\hline С38-С37-Н37А & 108.7 \\
\hline С36-С37-Н37В & 108.7 \\
\hline С38-С37-Н37В & 108.7 \\
\hline Н37А-С37-Н37В & 107.6 \\
\hline N39-C38-C37 & $110.9(2)$ \\
\hline N39-C38-H38A & 109.5 \\
\hline С37-С38-H38А & 109.5 \\
\hline N39-C38-H38B & 109.5 \\
\hline С37-С38-Н38В & 109.5 \\
\hline Н38А-С38-Н38В & 108.1 \\
\hline C2-N3-N4 & 112.73(19) \\
\hline C2-N3-H3 & 123.6 \\
\hline N4-N3-H3 & 123.6 \\
\hline C5-N4-N3 & $102.2(2)$ \\
\hline C8-N7-C5 & $125.5(2)$ \\
\hline C8-N7-H7 & 117.2 \\
\hline C5-N7-H7 & 117.2 \\
\hline C11-N10-C9 & $123.9(2)$ \\
\hline C11-N10-H10 & 118.1 \\
\hline C9-N10-H10 & 118.1 \\
\hline C14-N13-C12 & $120.3(2)$ \\
\hline C14-N13-H13 & 119.8 \\
\hline C12-N13-H13 & 119.8 \\
\hline N17-N16-C15 & 111.36(18) \\
\hline N17-N16-H16 & 124.3 \\
\hline C15-N16-H16 & 124.3 \\
\hline C18-N17-N16 & 101.65(18) \\
\hline C21-N20-C18 & $122.7(2)$ \\
\hline C21-N20-H20 & 118.6 \\
\hline C18-N20-H20 & 118.6 \\
\hline C38-N39-H39A & 109.5 \\
\hline C38-N39-H39B & 109.5 \\
\hline H39A-N39-H39B & 109.5 \\
\hline
\end{tabular}


C38-N39-H39C

H39A-N39-H39C

H39B-N39-H39C

C1-O2-C31

109.5

109.5

109.5

114.5(2)
C22-O23-C24

C25-O26-C27

C30-O29-C28
113.87(19)

110.9(2)

109.6(2)

Symmetry transformations used to generate equivalent atoms: 
Table S4. Anisotropic displacement parameters $\left(\AA^{2}\right)$ for $\mathbf{1}$. The anisotropic displacement factor exponent takes the form: $-2 \pi^{2}\left[h^{2} a^{* 2} U^{11}+\ldots+2 h k a^{*} b^{*} U^{12}\right]$

\begin{tabular}{|c|c|c|c|c|c|c|}
\hline & $\mathrm{U}^{11}$ & $\mathrm{U}^{22}$ & $\mathrm{U}^{33}$ & $\mathrm{U}^{23}$ & $\mathrm{U}^{13}$ & $\mathrm{U}^{12}$ \\
\hline C1 & $0.093(2)$ & $0.083(2)$ & $0.114(2)$ & $0.0052(15)$ & $-0.0006(17)$ & $0.0426(19)$ \\
\hline C2 & $0.091(2)$ & $0.0817(18)$ & $0.0869(17)$ & $-0.0020(13)$ & $0.0022(14)$ & $0.0553(18)$ \\
\hline C5 & $0.0886(19)$ & $0.089(2)$ & $0.0718(16)$ & $-0.0070(13)$ & $0.0012(13)$ & $0.0550(17)$ \\
\hline C6 & $0.0595(16)$ & $0.0764(17)$ & $0.1020(19)$ & $-0.0024(14)$ & $0.0002(13)$ & $0.0296(14)$ \\
\hline C8 & $0.095(2)$ & $0.100(2)$ & $0.0688(16)$ & $0.0085(14)$ & $0.0115(14)$ & $0.0606(18)$ \\
\hline C9 & $0.0691(16)$ & $0.0731(16)$ & $0.1034(19)$ & $-0.0017(15)$ & $0.0160(13)$ & $0.0316(14)$ \\
\hline C11 & $0.0606(15)$ & $0.102(2)$ & $0.094(2)$ & $0.0063(16)$ & $-0.0089(14)$ & $0.0360(14)$ \\
\hline C12 & $0.0661(15)$ & $0.0717(16)$ & $0.108(2)$ & $-0.0084(14)$ & $-0.0199(14)$ & $0.0282(13)$ \\
\hline C14 & $0.0642(16)$ & $0.0757(18)$ & $0.0825(18)$ & $-0.0038(14)$ & $0.0010(12)$ & $0.0258(16)$ \\
\hline C15 & $0.0672(16)$ & $0.0737(17)$ & $0.0843(17)$ & $-0.0036(13)$ & $0.0057(12)$ & $0.0320(15)$ \\
\hline C18 & $0.0861(19)$ & $0.0843(18)$ & $0.0813(15)$ & $-0.0013(13)$ & $-0.0074(14)$ & $0.0537(18)$ \\
\hline C19 & $0.0808(18)$ & $0.0652(15)$ & $0.0823(16)$ & $-0.0014(12)$ & $0.0000(12)$ & $0.0407(14)$ \\
\hline C21 & $0.0701(17)$ & $0.0726(19)$ & $0.098(2)$ & $-0.0089(14)$ & -0.0023 & $0.0287(15)$ \\
\hline C22 & $0.0864(18)$ & $0.094(2)$ & $0.0920(18)$ & $-0.0028(15)$ & $-0.0143(14)$ & $0.0450(16)$ \\
\hline C24 & $0.102(2)$ & $0.0786(19)$ & $0.171(3)$ & $0.0088(18)$ & $-0.0093(19)$ & $0.0626(17)$ \\
\hline C25 & $0.120(2)$ & $0.093(2)$ & $0.172(3)$ & $0.013(2)$ & $0.021(2)$ & $0.074(2)$ \\
\hline C27 & $0.138(3)$ & $0.079(2)$ & $0.125(2)$ & $-0.0071(17)$ & $-0.014(2)$ & $0.0553(19)$ \\
\hline C28 & $0.111(2)$ & $0.0868(19)$ & $0.127(2)$ & $0.0261(17)$ & $-0.002(2)$ & $0.0392(19)$ \\
\hline C30 & $0.114(3)$ & $0.146(3)$ & $0.233(4)$ & 0.063(3) & $0.029(3)$ & $0.057(3)$ \\
\hline C31 & $0.099(2)$ & 0.062 & $0.404(7)$ & & & $0.0409(17)$ \\
\hline C32 & $0.0836(17)$ & $0.0832(17)$ & $0.0961(18)$ & 0.0022 & 0.013 & $0.0259(15)$ \\
\hline C33 & $0.096(2)$ & $0.0815(19)$ & $0.173(3)$ & $0.0053(19)$ & $0.042(2)$ & $0.0151(16)$ \\
\hline C34 & $0.146(3)$ & $0.0903(19)$ & 0.0951(19) & $-0.0021(16)$ & $0.0164(18)$ & $0.0495(19)$ \\
\hline C35 & $0.0812(17)$ & $0.0871(19)$ & $0.114(2)$ & $0.0111(15)$ & $-0.0120(15)$ & $0.0347(15)$ \\
\hline C36 & $0.0785(17)$ & $0.101(2)$ & $0.104(2)$ & $-0.0013(16)$ & $-0.0076(15)$ & $0.0348(15)$ \\
\hline C37 & 0.0821(17) & $0.113(2)$ & $0.0906(19)$ & $0.0128(17)$ & $0.0025(14)$ & $0.0427(16)$ \\
\hline C38 & $0.0851(18)$ & $0.0918(19)$ & $0.114(2)$ & $0.0257(17)$ & $0.0070(16)$ & $0.0380(15)$ \\
\hline N3 & $0.0689(13)$ & $0.0923(16)$ & $0.0837(12)$ & $-0.0026(11)$ & $0.0059(10)$ & $0.0459(13)$ \\
\hline N4 & $0.0614(12)$ & $0.0640(13)$ & $0.0867(13)$ & $0.0073(10)$ & $0.0078(10)$ & $0.0243(11)$ \\
\hline N7 & 0.0794(15) & $0.0550(12)$ & $0.1053(15)$ & $-0.0058(10)$ & $0.0011(12)$ & $0.0278(12)$ \\
\hline N10 & $0.0640(12)$ & $0.0904(14)$ & $0.1097(16)$ & $-0.0046(14)$ & $0.0020(11)$ & $0.0393(11)$ \\
\hline N13 & $0.0719(14)$ & $0.0801(14)$ & $0.1036(16)$ & $-0.0022(11)$ & $-0.0007(11)$ & $0.0358(12)$ \\
\hline N16 & $0.0793(14)$ & $0.0643(12)$ & $0.0783(13)$ & $-0.0046(10)$ & $-0.0069(10)$ & $0.0411(12)$ \\
\hline N17 & $0.0686(13)$ & $0.0682(13)$ & $0.0778(11)$ & $-0.0049(9)$ & $0.0005(10)$ & $0.0322(11)$ \\
\hline N20 & $0.0870(14)$ & $0.0742(15)$ & $0.0745(13)$ & $-0.0129(11)$ & $0.0055(11)$ & $0.0337(12)$ \\
\hline N39 & $0.0939(15)$ & $0.1053(16)$ & $0.1030(15)$ & $0.0099(13)$ & $-0.0102(12)$ & $0.0555(13)$ \\
\hline $\mathrm{O} 1$ & 0.0753(12) & 0.0788(11) & 0.1073(13) & $-0.0050(9)$ & $-0.0055(10)$ & $0.0353(10)$ \\
\hline $\mathrm{O} 2$ & $0.0693(11)$ & $0.0691(11)$ & $0.225(2)$ & $-0.0188(12)$ & $-0.0176(12)$ & $0.0362(10)$ \\
\hline $\mathrm{O} 3$ & $0.0697(10)$ & $0.0861(12)$ & $0.1248(14)$ & $-0.0038(10)$ & $0.0060(9)$ & $0.0422(10)$ \\
\hline $\mathrm{O} 4$ & $0.0830(12)$ & $0.1087(14)$ & $0.1299(15)$ & $0.0000(11)$ & $0.0016(10)$ & $0.0538(11)$ \\
\hline O5 & 0.0761(11) & $0.0761(12)$ & 0.1133(13) & 0.0016(9) & 0.0031(9) & $0.0432(10)$ \\
\hline O6 & 0.0954(14) & $0.0900(13)$ & $0.1224(14)$ & $-0.0065(10)$ & $-0.0054(11)$ & $0.0506(12)$ \\
\hline $\mathrm{O} 23$ & & & & $-0.0095(10)$ & $-0.0024(10)$ & $0.0415(10)$ \\
\hline $\mathrm{O} 26$ & 0.1193(15) & $0.0806(12)$ & $0.1051(13)$ & $0.0101(10)$ & $0.0096(11)$ & $0.0572(11)$ \\
\hline O29 & $0.1232(16)$ & $0.0877(13)$ & $0.1101(13)$ & $0.0318(10)$ & $0.0021(12)$ & $0.0436(13)$ \\
\hline
\end{tabular}


Table S5. Hydrogen coordinates and isotropic displacement parameters $\left(\AA^{2}\right)$ for $\mathbf{1}$.

\begin{tabular}{|c|c|c|c|c|c|}
\hline & $\mathrm{x}$ & $\mathrm{y}$ & $\mathrm{z}$ & $\mathrm{U}(\mathrm{eq})$ & Occupancy \\
\hline H6 & 0.8735 & 0.0632 & 0.1468 & 0.097 & 1 \\
\hline H9 & 0.7218 & -0.0944 & 0.1485 & 0.100 & 1 \\
\hline H12 & 0.7425 & -0.1264 & 0.0245 & 0.102 & 1 \\
\hline H19 & 0.8581 & -0.1857 & 0.0478 & 0.089 & 1 \\
\hline H22A & 1.0061 & -0.2062 & 0.0369 & 0.109 & 1 \\
\hline Н22B & 0.9880 & -0.2209 & 0.0799 & 0.109 & 1 \\
\hline H24A & 0.9880 & -0.2972 & 0.0823 & 0.131 & 1 \\
\hline H24B & 1.0177 & -0.2790 & 0.0428 & 0.131 & 1 \\
\hline H25A & 0.9463 & -0.3512 & 0.0129 & 0.143 & 1 \\
\hline H25B & 0.9781 & -0.3657 & 0.0407 & 0.143 & 1 \\
\hline H27A & 0.9124 & -0.4445 & 0.0543 & 0.136 & 1 \\
\hline H27B & 0.8794 & -0.4352 & 0.0238 & 0.136 & 1 \\
\hline H28A & 0.8283 & -0.4964 & 0.0719 & 0.136 & 1 \\
\hline H28B & 0.8524 & -0.4524 & 0.1029 & 0.136 & 1 \\
\hline H30A & 0.7472 & -0.5049 & 0.0778 & 0.252 & 1 \\
\hline Н30В & 0.7441 & -0.4549 & 0.0812 & 0.252 & 1 \\
\hline Н30С & 0.7731 & -0.4678 & 0.1127 & 0.252 & 1 \\
\hline H31A & 1.0113 & 0.2220 & 0.1352 & 0.282 & 1 \\
\hline Н31В & 0.9889 & 0.2257 & 0.1751 & 0.282 & 1 \\
\hline Н31С & 0.9602 & 0.2232 & 0.1367 & 0.282 & 1 \\
\hline H32 & 0.7770 & -0.1345 & 0.1856 & 0.114 & 1 \\
\hline Н33А & 0.6940 & -0.1991 & 0.2070 & 0.194 & 1 \\
\hline Н33В & 0.6992 & -0.1996 & 0.1619 & 0.194 & 1 \\
\hline Н33С & 0.6695 & -0.1749 & 0.1807 & 0.194 & 1 \\
\hline H34A & 0.7866 & -0.0745 & 0.2259 & 0.172 & 1 \\
\hline Н34В & 0.7381 & -0.1224 & 0.2424 & 0.172 & 1 \\
\hline Н34C & 0.7324 & -0.0823 & 0.2174 & 0.172 & 1 \\
\hline H35A & 0.6930 & -0.2147 & 0.0697 & 0.117 & 1 \\
\hline Н35В & 0.7017 & -0.2157 & 0.0252 & 0.117 & 1 \\
\hline H36A & 0.6203 & -0.2332 & 0.0358 & 0.119 & 1 \\
\hline Н36В & 0.6394 & -0.1836 & 0.0603 & 0.119 & 1 \\
\hline H37A & 0.6697 & -0.1360 & 0.0071 & 0.117 & 1 \\
\hline Н37В & 0.6112 & -0.1747 & 0.0036 & 0.117 & 1 \\
\hline H38A & 0.6824 & -0.1959 & -0.0292 & 0.120 & 1 \\
\hline Н38В & 0.6237 & -0.2236 & -0.0381 & 0.120 & 1 \\
\hline H3 & 0.9971 & 0.0597 & 0.1352 & 0.095 & 1 \\
\hline H7 & 0.8426 & -0.0689 & 0.1392 & 0.099 & 1 \\
\hline H10 & 0.7643 & -0.1444 & 0.1155 & 0.105 & 1 \\
\hline H13 & 0.7852 & -0.1771 & 0.0512 & 0.103 & 1 \\
\hline H16 & 0.9283 & -0.0391 & 0.0472 & 0.086 & 1 \\
\hline H20 & 0.9951 & -0.1370 & 0.0459 & 0.098 & 1 \\
\hline H39A & 0.6423 & -0.1420 & -0.0608 & 0.147 & 1 \\
\hline Н39В & 0.6566 & -0.1713 & -0.0860 & 0.147 & 1 \\
\hline Н39С & 0.6952 & -0.1301 & -0.0626 & 0.147 & 1 \\
\hline
\end{tabular}


Table S6. Torsion angles $\left[^{\circ}\right]$ for $\mathbf{1}$.

\begin{tabular}{|c|c|c|c|}
\hline O1-C1-C2-N3 & $10.3(4)$ & С35-С36-С37-С38 & 60.1(3) \\
\hline O2-C1-C2-N3 & $-173.9(2)$ & C36-C37-C38-N39 & $-165.8(2)$ \\
\hline O1-C1-C2-C6 & $-170.1(3)$ & C6-C2-N3-N4 & $-1.6(3)$ \\
\hline O2-C1-C2-C6 & $5.7(4)$ & C1-C2-N3-N4 & $178.0(2)$ \\
\hline N3-C2-C6-C5 & 2.7(3) & C6-C5-N4-N3 & $2.2(3)$ \\
\hline C1-C2-C6-C5 & $-176.8(2)$ & N7-C5-N4-N3 & 177.69(18) \\
\hline N4-C5-C6-C2 & $-3.2(3)$ & C2-N3-N4-C5 & $-0.4(2)$ \\
\hline N7-C5-C6-C2 & $-178.0(2)$ & O3-C8-N7-C5 & $-0.9(4)$ \\
\hline O3-C8-C9-N10 & $130.7(2)$ & C9-C8-N7-C5 & $179.6(2)$ \\
\hline N7-C8-C9-N10 & $-49.9(3)$ & N4-C5-N7-C8 & $176.1(2)$ \\
\hline O3-C8-C9-C32 & $-103.0(3)$ & C6-C5-N7-C8 & $-9.2(4)$ \\
\hline N7-C8-C9-C32 & $76.4(3)$ & O4-C11-N10-C9 & $-7.3(4)$ \\
\hline O4-C11-C12-N13 & $148.2(2)$ & C12-C11-N10-C9 & $178.4(2)$ \\
\hline N10-C11-C12-N13 & $-37.4(3)$ & C8-C9-N10-C11 & $-80.9(3)$ \\
\hline O4-C11-C12-C35 & $-88.7(3)$ & C32-C9-N10-C11 & $153.0(2)$ \\
\hline N10-C11-C12-C35 & $85.7(3)$ & O5-C14-N13-C12 & $4.8(4)$ \\
\hline O5-C14-C15-C19 & 179.2(3) & C15-C14-N13-C12 & $-176.0(2)$ \\
\hline N13-C14-C15-C19 & $0.0(4)$ & C11-C12-N13-C14 & $-62.8(3)$ \\
\hline O5-C14-C15-N16 & $0.8(4)$ & C35-C12-N13-C14 & $174.0(2)$ \\
\hline N13-C14-C15-N16 & $-178.38(19)$ & C19-C15-N16-N17 & 1.1(3) \\
\hline N17-C18-C19-C15 & $0.6(3)$ & C14-C15-N16-N17 & 179.94(19) \\
\hline N20-C18-C19-C15 & $178.5(2)$ & C19-C18-N17-N16 & $0.1(2)$ \\
\hline N16-C15-C19-C18 & $-1.0(3)$ & N20-C18-N17-N16 & $-178.18(17)$ \\
\hline C14-C15-C19-C18 & $-179.5(3)$ & C15-N16-N17-C18 & $-0.7(2)$ \\
\hline O6-C21-C22-O23 & 19.6(4) & O6-C21-N20-C18 & $4.5(4)$ \\
\hline N20-C21-C22-O23 & $-162.8(2)$ & C22-C21-N20-C18 & $-173.1(2)$ \\
\hline O23-C24-C25-O26 & $-72.3(3)$ & C19-C18-N20-C21 & $-0.4(3)$ \\
\hline O26-C27-C28-O29 & 68.3(3) & N17-C18-N20-C21 & $177.5(2)$ \\
\hline N10-C9-C32-C34 & $175.9(2)$ & O1-C1-O2-C31 & $3.2(4)$ \\
\hline C8-C9-C32-C34 & $50.6(3)$ & C2-C1-O2-C31 & $-172.7(3)$ \\
\hline N10-C9-C32-C33 & $-60.0(3)$ & C21-C22-O23-C24 & $-169.9(2)$ \\
\hline C8-C9-C32-C33 & $174.7(2)$ & C25-C24-O23-C22 & $-176.1(2)$ \\
\hline N13-C12-C35-C36 & $-171.2(2)$ & C24-C25-O26-C27 & $-173.8(2)$ \\
\hline C11-C12-C35-C36 & 65.2(3) & C28-C27-O26-C25 & $-179.9(2)$ \\
\hline C12-C35-C36-C37 & $55.6(4)$ & С27-C28-O29-С30 & 178.0(3) \\
\hline
\end{tabular}

Symmetry transformations used to generate equivalent atoms:

Table S7. Hydrogen bonds for aminopyrazole hybrid peptide 1 [ $\AA$ and ${ }^{\circ}$ ].

\begin{tabular}{lcccc}
\hline D-H...A & d(D-H) & $d(H . . A)$ & $d(D . . A)$ & $<($ DHA $)$ \\
\hline N3-H3...N4\#1 & 0.88 & 2.16 & $3.021(3)$ & 165.8 \\
N16-H16...N17\#1 & 0.88 & 2.15 & $3.017(2)$ & 167.0 \\
N7-H7...O1\#2 & 0.88 & 1.99 & $2.861(3)$ & 172.2 \\
N10-H10...O1\#2 & 0.88 & 2.40 & $3.051(3)$ & 131.2 \\
N20-H20...O5\#2 & 0.88 & 1.85 & $2.724(3)$ & 174.0 \\
N39-H39C...O6\#3 & 0.91 & 1.95 & $2.787(3)$ & 151.3 \\
N39-H39C...O23\#3 & 0.91 & 2.39 & $3.026(3)$ & 126.5 \\
N39-H39A...O26\#3 & 0.91 & 2.14 & $2.999(3)$ & 157.9 \\
N39-H39A...O29\#3 & 0.91 & 2.32 & $2.907(3)$ & 122.4
\end{tabular}

Symmetry transformations used to generate equivalent atoms:

$\# 1-x+y+2,-x+1, z \quad \# 2-y+1, x-y-1, z \quad \# 3$ y+1,x-1,-z 


\section{NMR spectroscopy:}

$20 \mathrm{mg} \mathrm{PzKVPz}$ in $0.6 \mathrm{~mL} \mathrm{H} 20 / \mathrm{D} 20(9: 1)$ at $298 \mathrm{~K}$
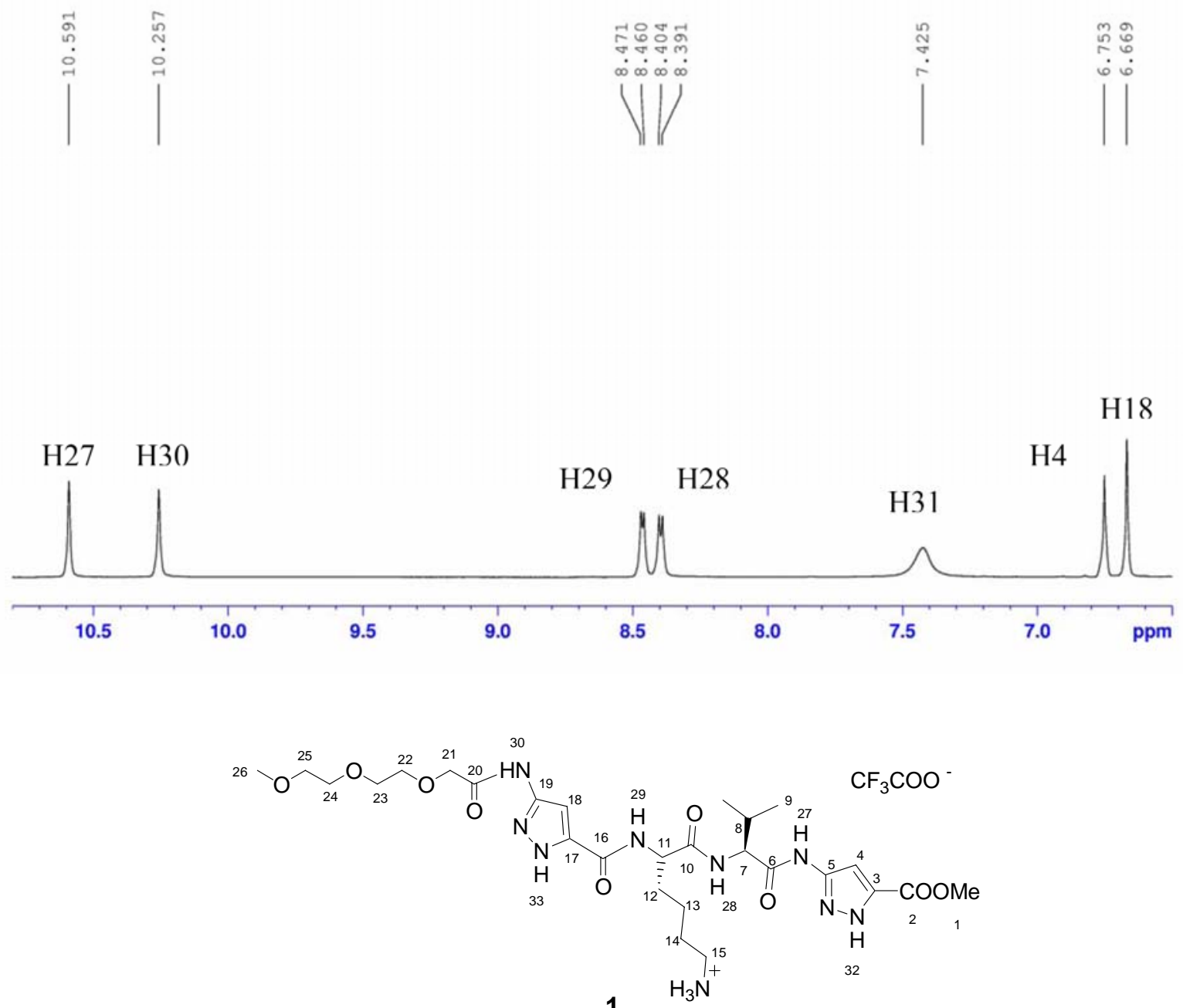

1

31

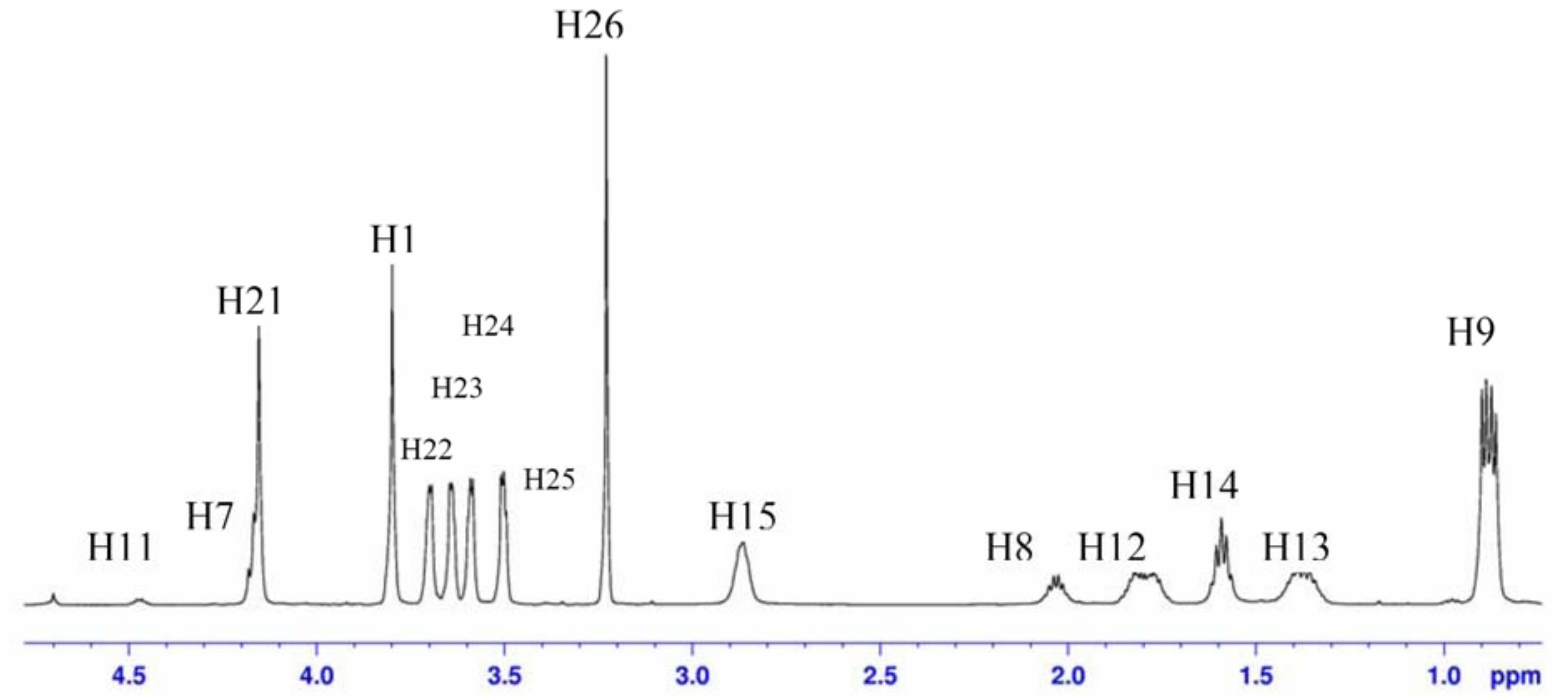

Figure S7. ${ }^{1} \mathrm{H}$ NMR spectra of 1 with assignments: Monomer at $298 \mathrm{~K}$. Calibration was carried out after FID aquisition with d8-THF set to $1.73 \mathrm{ppm}$. 

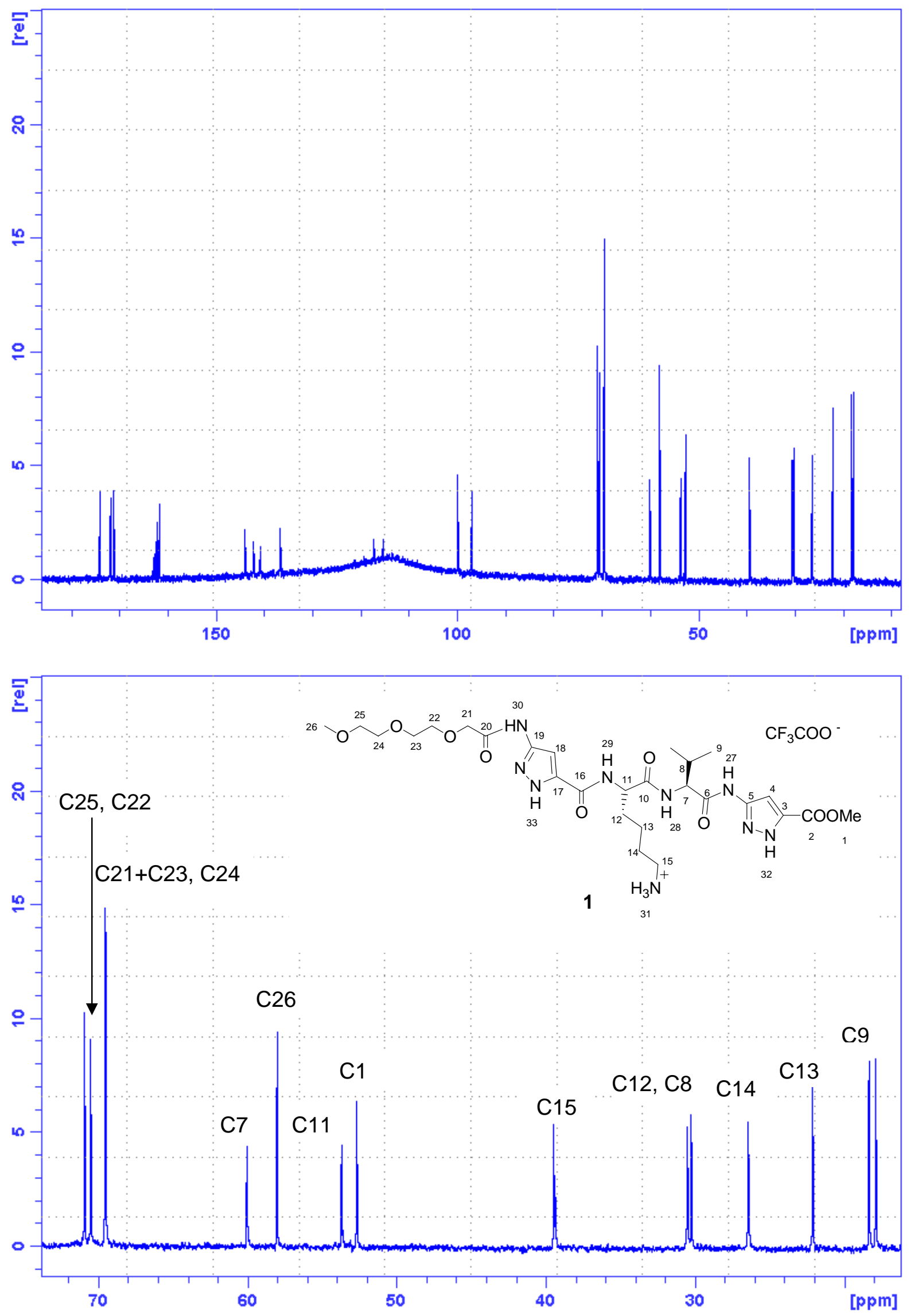

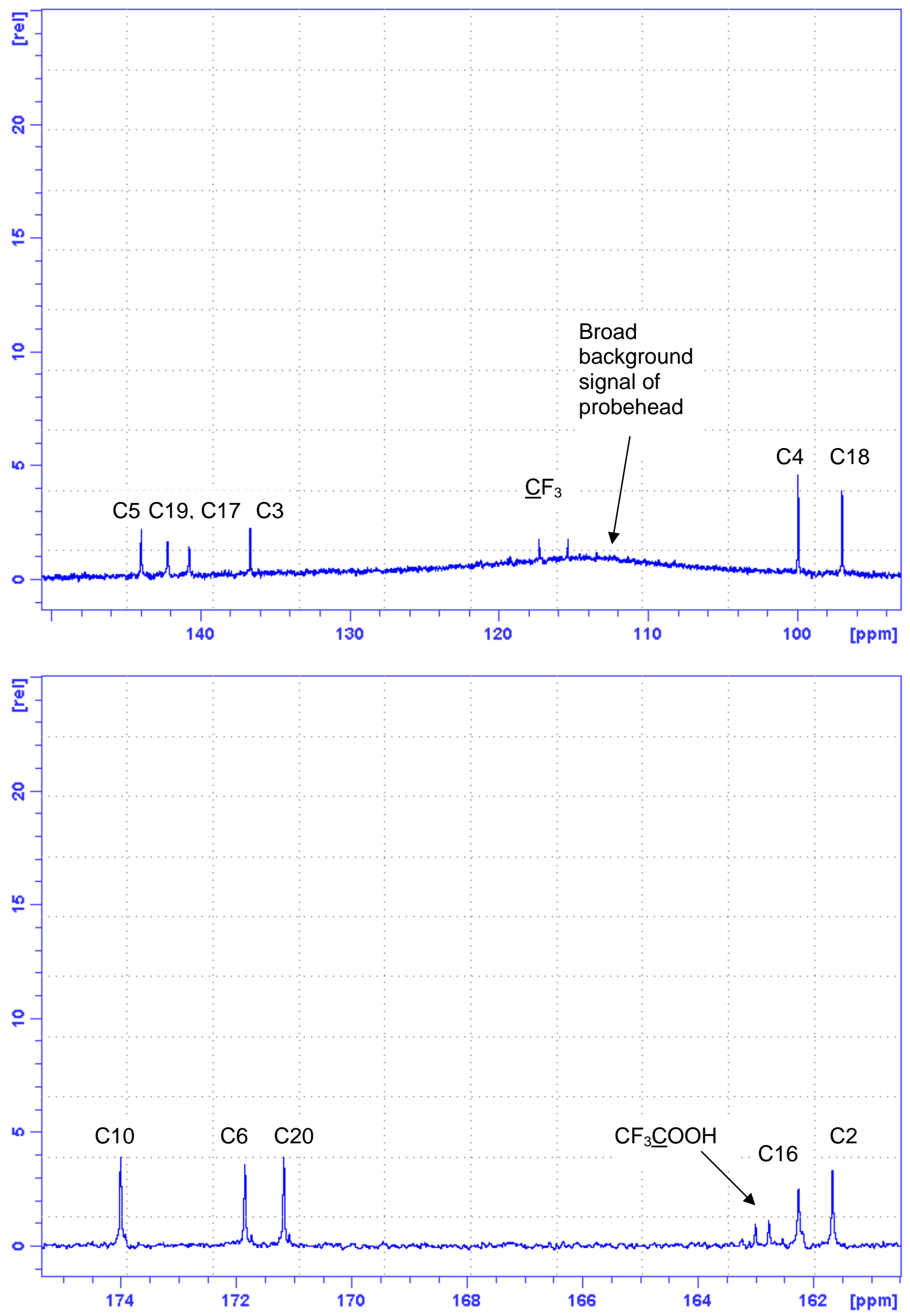

Figure S8. ${ }^{13} \mathrm{C}$ NMR spectrum of $\mathbf{1}$ in $\mathrm{H}_{2} \mathrm{O} / \mathrm{D}_{2} \mathrm{O}$ (9:1) at $298 \mathrm{~K}$ : a) overview; b) 0-74 ppm; c) 94-150 ppm; d) 161-175 ppm. The assignment is based on ${ }^{1} \mathrm{H}$, DQF-COSY, HSQC, and HMBC spectra. 


$$
26 \overbrace{24}^{25}
$$

1

31

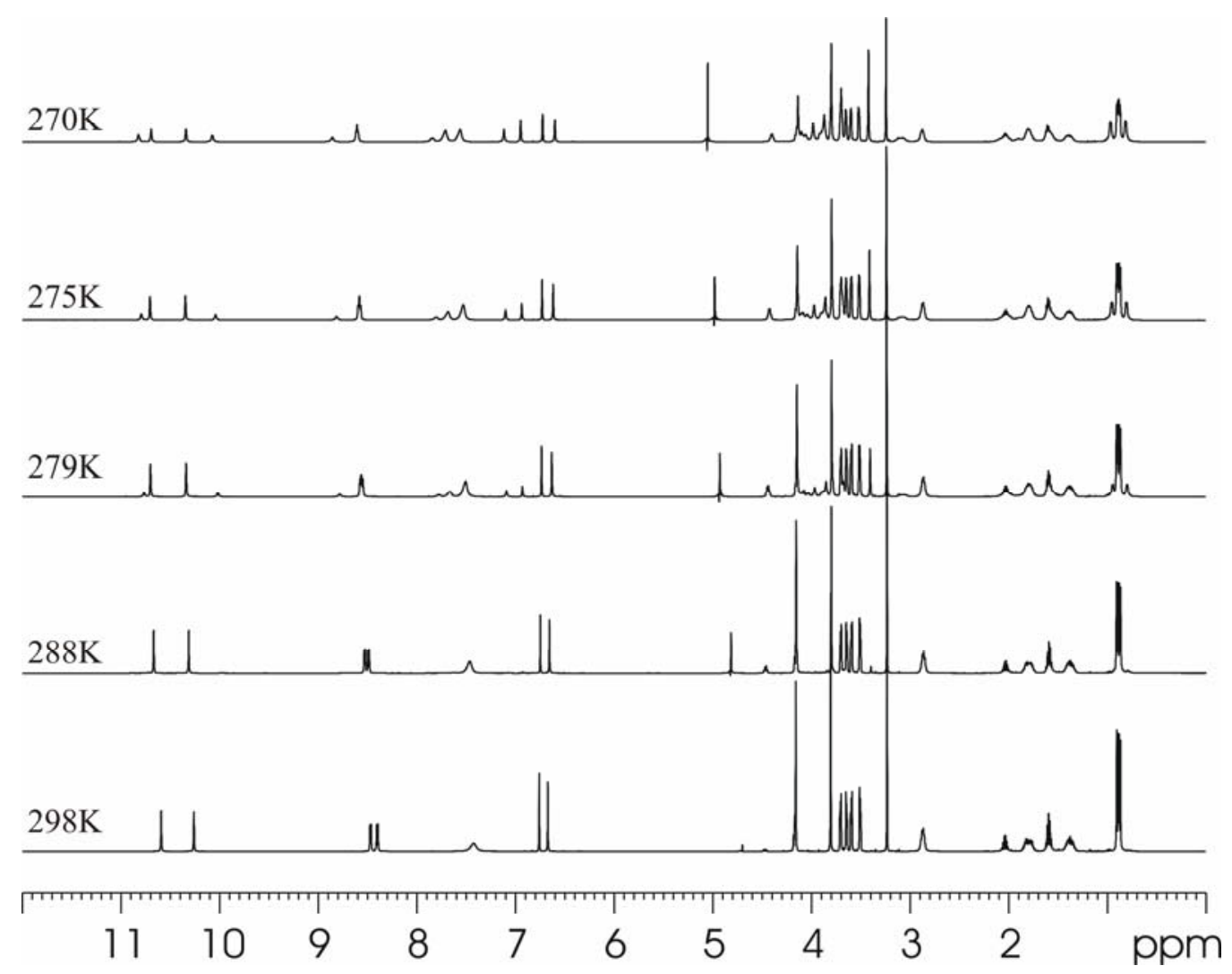

Figure S9. Variable temperature experiment $\left({ }^{1} \mathrm{H}\right.$ NMR) of a $20 \mathrm{mM}$ solution of 1 in $\mathrm{H}_{2} \mathrm{O}: \mathrm{D}_{2} \mathrm{O}$ (9:1). Note the increasing new set of signals below $280 \mathrm{~K}$. Calibration was carried out with d8-THF set to $1.73 \mathrm{ppm}$. 

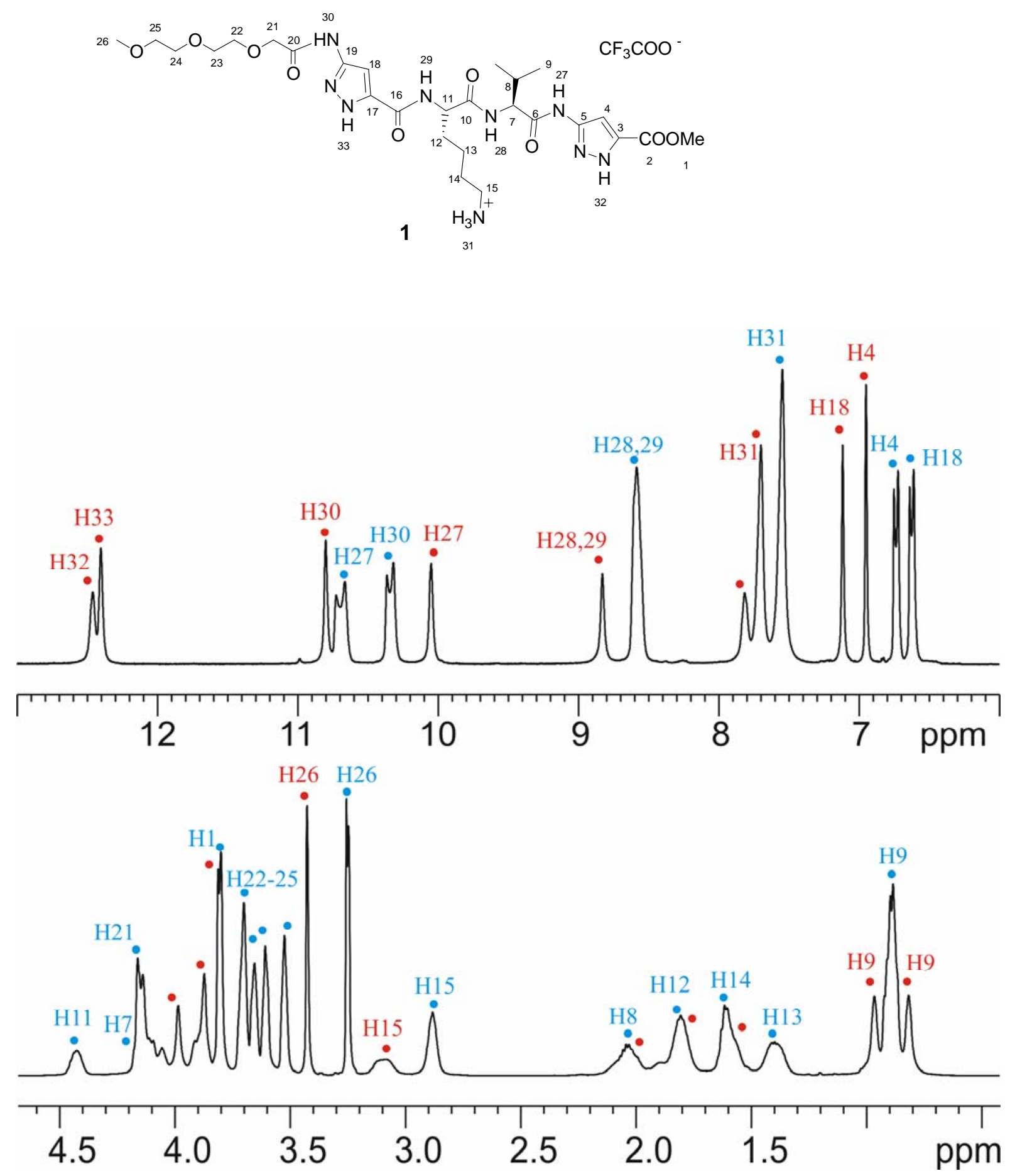

Figure S10. ${ }^{1}$ H NMR spectra with assignments: Monomer (light blue) and Hexamer (red) at $275 \mathrm{~K}$. 
ROESY (overview):

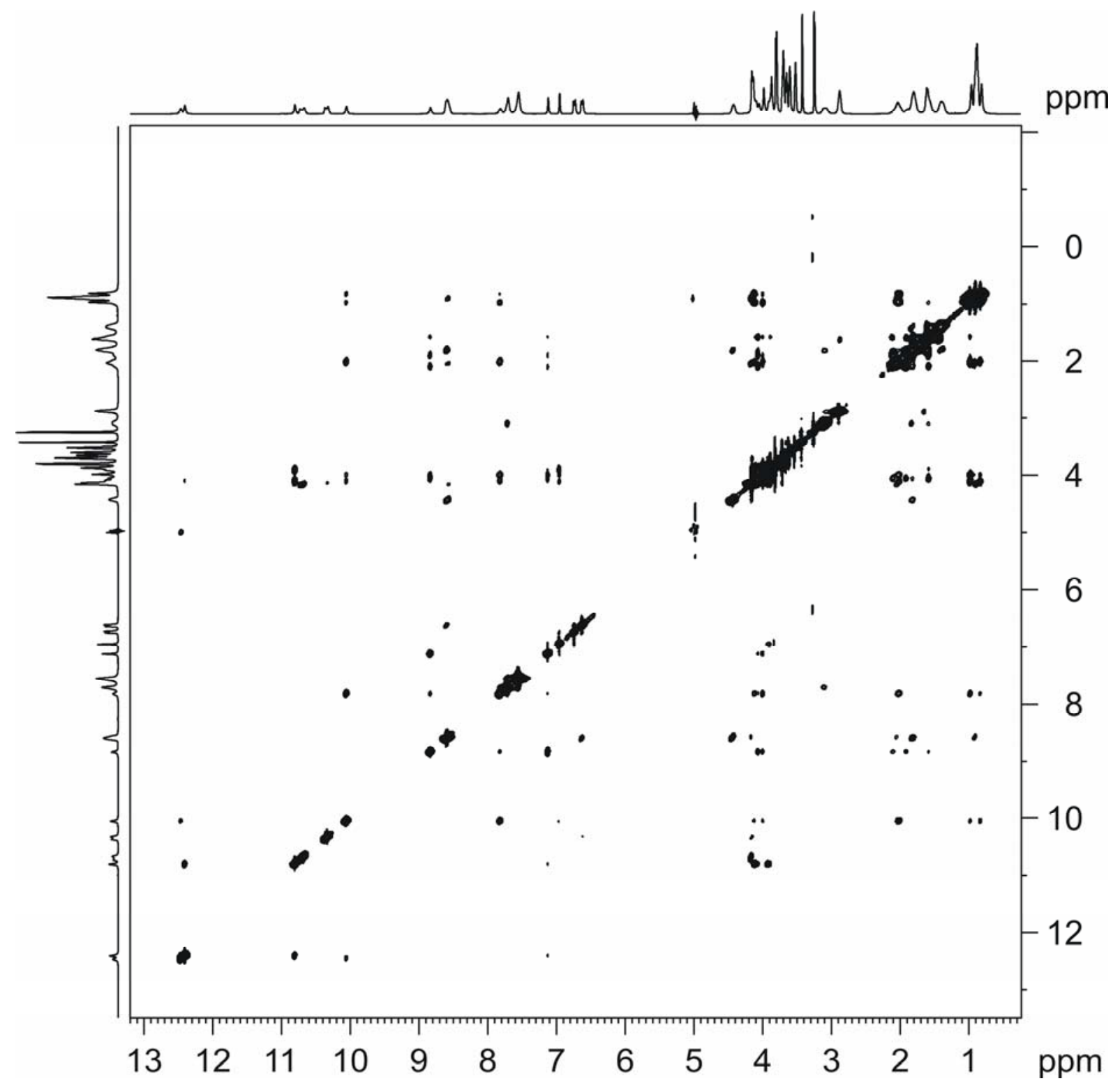

Figure S11. ROESY spectrum of 1 in water at 275 K. Mixing time 150 ms.

\section{Detailed analysis (see next page):}

Intra-molecular crosspeaks (black numbers) in monomer (blue circle, 21-30, 18-29, 29-27) and/or aggregate (red circle, 12-15, 21-30, 29-27, 29-28) demonstrate conformational pre- or reorganisation. (Correlations and Tables see 2 pages further).

Inter-molecular crosspeaks (red numbers) in the complex (red circles, 21-14, 15-31, 21-27, 21-4, 4-30, 27-32, 30-33) prove self-assembly and support hexameric structure analogous to the crystal structure. (Correlations and Tables see 2 pages further). 
ROESY (detailed analysis):
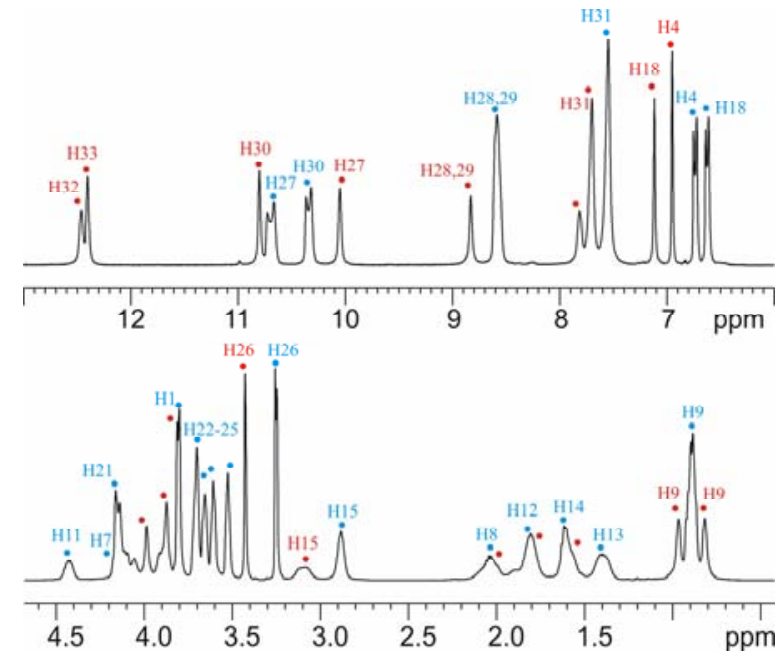
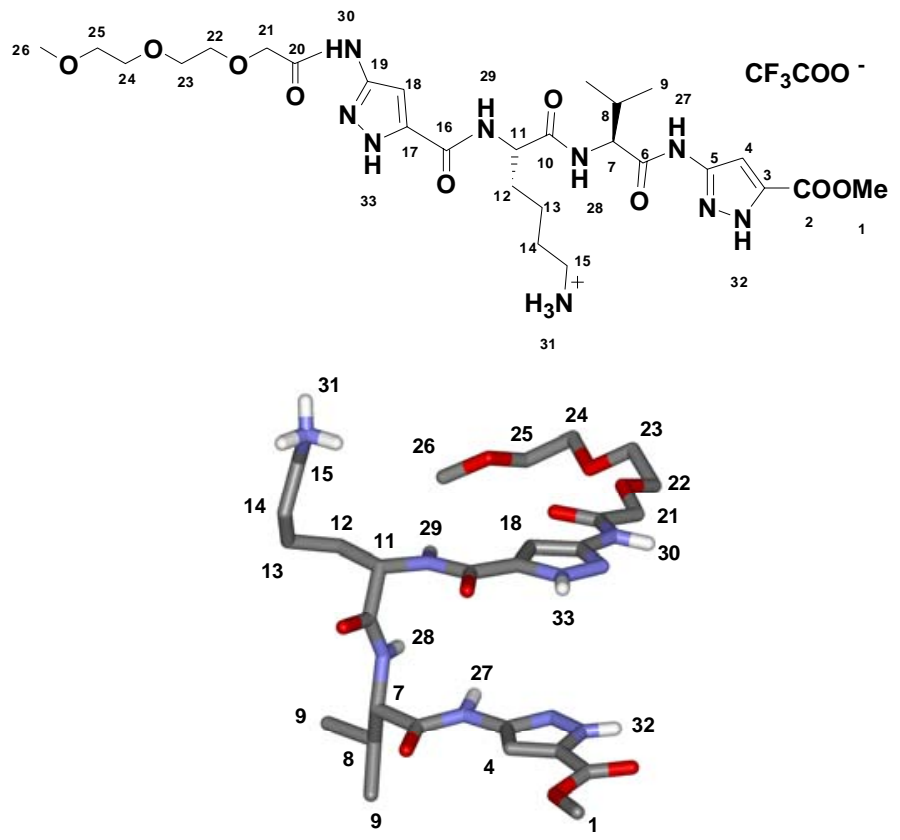

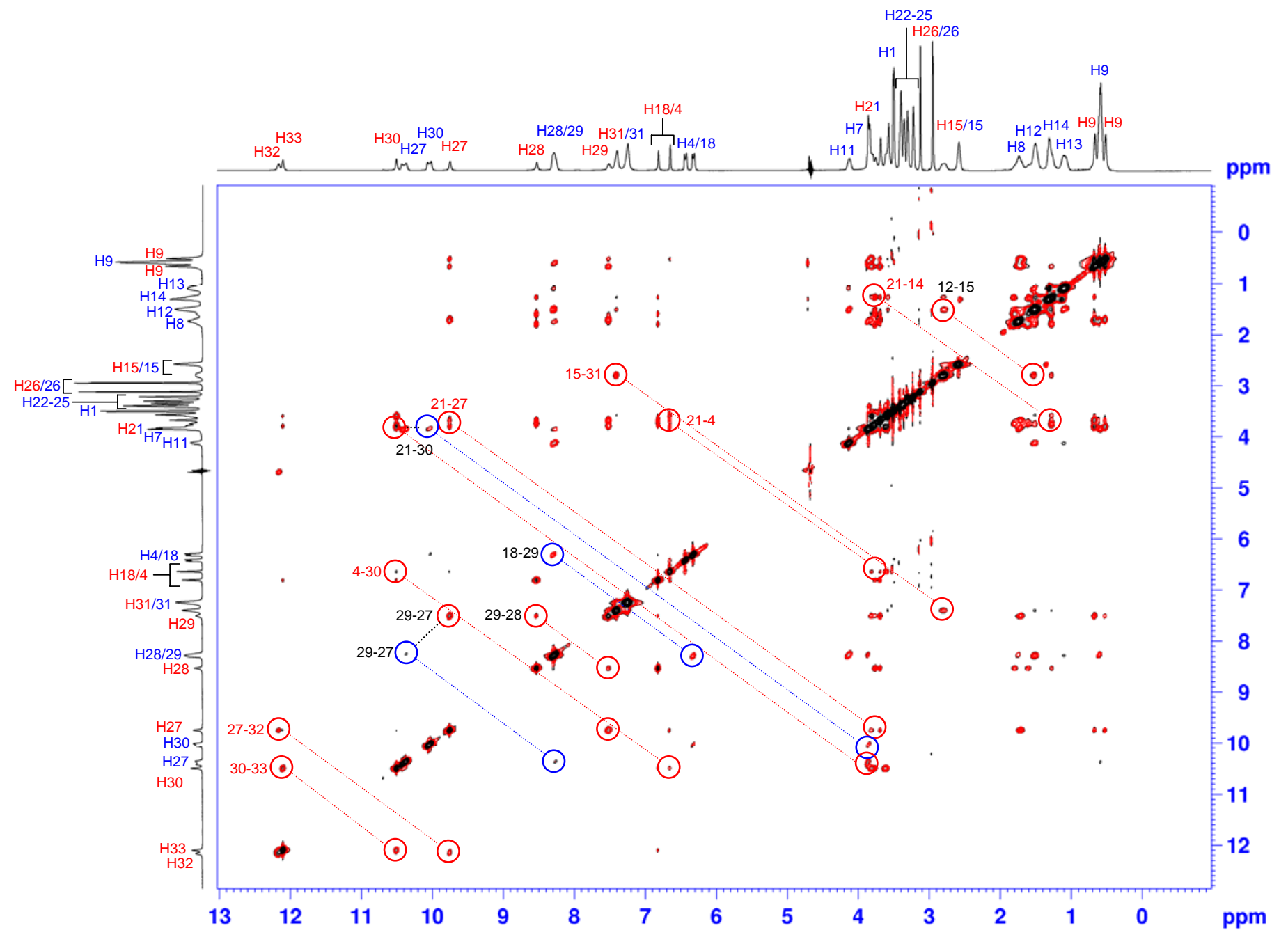

Legend:29-28 black numbers: intra-molecular crosspeak 27-32 red numbers: inter-molecular crosspeak

$\bigcirc$ blue circle: monomer signals

$\bigcirc$ red circles: hexamer signals 
Monomer: some characteristic intra-molecular distances (X-Ray) and ROEs*

\begin{tabular}{|c|c|c|}
\hline Atoms & $\mathrm{R}_{\mathrm{HH}}(\AA)$ & ROE \\
\hline $\mathrm{H}_{28}-\mathrm{H}_{29}$ & 2.69 & strong \\
\hline $\mathrm{H}_{27}-\mathrm{H}_{4}$ & 3.74 & strong \\
\hline $\mathrm{H}_{29}-\mathrm{H}_{18}$ & 2.42 & strong \\
\hline $\mathrm{H}_{30}-\mathrm{H}_{18}$ & 3.66 & strong \\
\hline $\mathrm{H}_{21}-\mathrm{H}_{30}$ & & strong \\
\hline $\mathrm{H}_{27}-\mathrm{H}_{29}$ & 3.72 & medium \\
\hline
\end{tabular}

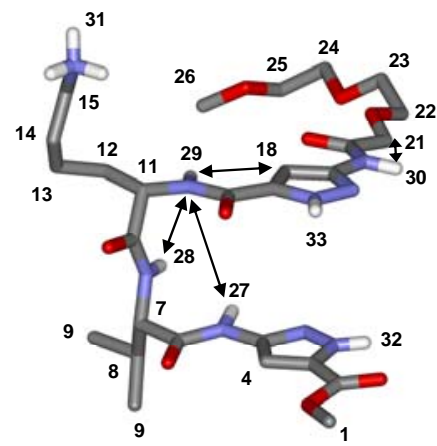

Intra-molecular crosspeaks which demonstrate preorganization: Valine's and lysine's amide protons $\mathrm{H}_{28}-\mathrm{H}_{29}$ in close vicinity marks the second kink in the peptide's backbone; the first was proven by Karplus analysis in water (kink at valine with $\theta=138^{\circ}$ ). Crosspeak $\mathrm{H}_{18}-\mathrm{H}_{29}$ between pyrazole- $\mathrm{CH}$ and lysine amide show the correct heterocycle orientation. Finally even a medium-size ROE contact is already established between $\mathrm{H}-27$ and $\mathrm{H}-29$, indicative a partial folding of the peptide into a coplanar arrangement of both heterocycles.

Hexamer: some characteristic inter-molecular distances (X-Ray) and ROEs*

\begin{tabular}{|c|c|c|}
\hline Atoms & $\mathrm{R}_{\mathrm{HH}}(\AA)$ & ROE \\
\hline $\mathrm{H}_{1}-\mathrm{H}_{8}$ & 3.09 & Not observed \\
\hline $\mathrm{H}_{1}-\mathrm{H}_{9}$ & 3.16 & Weak \\
\hline $\mathrm{H}_{1}-\mathrm{H}_{28}$ & 3.38 & Not observed \\
\hline $\mathrm{H}_{4}-\mathrm{H}_{21}$ & 3.21 & Strong \\
\hline $\mathrm{H}_{14}-\mathrm{H}_{23}$ & 3.05 & medium \\
\hline $\mathrm{H}_{11}-\mathrm{H}_{18}$ & 3.67 & medium \\
\hline $\mathrm{H}_{4}-\mathrm{H}_{30}$ & 3.85 & medium \\
\hline $\mathrm{H}_{25}-\mathrm{H}_{9}$ & 4.03 & Not observed \\
\hline $\mathrm{H}_{27}-\mathrm{H}_{32}$ & 2.85 & Strong \\
\hline $\mathrm{H}_{30}-\mathrm{H}_{33}$ & 2.84 & Strong \\
\hline
\end{tabular}

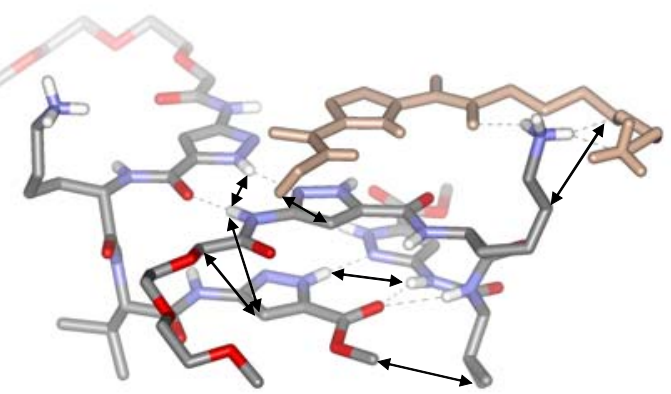

*ROESY spectrum was recorded at $275 \mathrm{~K}$.

Inter-molecular crosspeaks in the complex, which support the hexameric aggregate: these are all impossible in the monomeric state (distances $>4 \AA$ ). Pyrazole $\mathrm{NH}$ protons $\mathrm{H}_{27}-\mathrm{H}_{32}$ as well as $\mathrm{H}_{30}-\mathrm{H}_{33}$ can only be explained with rosette formation; they are supported by $\mathrm{H}_{1}-\mathrm{H}_{9}$. Neighbourhood of $\mathrm{H}_{4}-\mathrm{H}_{21}$ as well as $\mathrm{H}_{4}-\mathrm{H}_{30}$ proves the stacked arrangement, while ethyleneglycol's and lysine's $\mathrm{H}_{14}-\mathrm{H}_{23}$ pair indicate the halfcrown-arrangement.
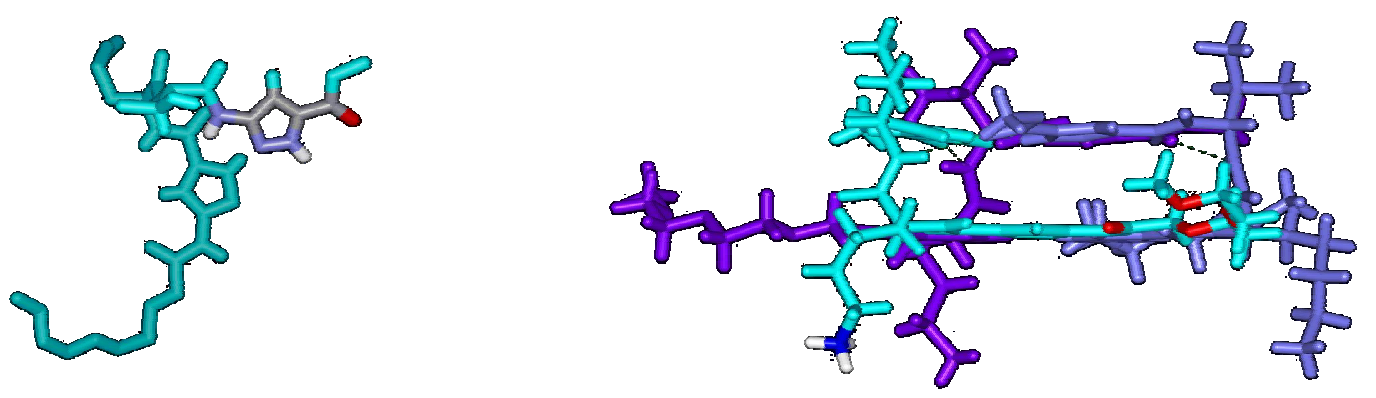
Counterargument 1: It might be argued that the NOEs ascribed to key intermolecular interaction H27-H32 and H30-H33 are likely due to intramolecular NOEs.

Response: The NOESY spectrum was recorded with a mixing time of $150 \mathrm{~ms}$ at $275 \mathrm{~K}$ (see Supporting Information Figure S11). The NOEs of H27-H32 and H30-H33 (pyrazole NH and pyrazole amide $\mathrm{NMH}$ ) were assigned to originate from intermolecular interactions, which are characteristic of the hexameric assembly. This was done on the basis of the following facts:

1) These cross peaks belong to proton signals of the hexamer (see Figures S10 and S11).

2) No NOEs were observed for the corresponding protons of monomer. This, however, may also be explained by the presence of the tautomeric effect in the monomer. Therefore,

3) We studied the NOE strength by calculating the volume integral of the NOESY cross peaks. The obtained volume integral of H30-H33 is stronger than that of H27-H32, which is in the range of that of $\mathrm{H} 27-\mathrm{H} 28$ (pyrazole amide $\mathrm{NH}$ and valine $\mathrm{NH}$ ) and about twice as strong as that of $\mathrm{H} 4-\mathrm{H} 27$ (pyrazole $\mathrm{CH}$ and pyrazole amide $\mathrm{NH}$ ). The X-ray structure revealed intramolecular distances of $4.3 \AA$ (H27-H32), $4.3 \AA$ (H30-H33), $3.7 \AA$ (H4-H27), and $2.6 \AA$ (H27-H28), whereas the intermolecular distances are $2.8 \AA$ (H27-H32) and also 2.8 $\AA$ (H30-H33). Therefore, if the cross peaks H27-H32 and H30-H33 were due to intramolecular interactions, they would be weaker than that of H4-H27. The observation that NOEs H27-H32 and H30-H33 are stronger than that of H4-H27 can thus only be explained by intermolecular interactions.

Counterargument 2: At $275 \mathrm{~K}$ that $\mathrm{H} 32$ and H33 do hop between the pyrazole nitrogens as a result of tautomerization. It has been shown by X-ray crystal analysis (A. Baldy, J. Elguero, R. Faure, M. Pierrot, E. J. J. Vincent, J. Am. Chem. Soc. 1985, 105, 5290 and J. A. S. Smith, B. Wehrle, F. Aguilar-Parrilla, H.-H. Limbach, M. de la Concepcion Foces-Foces,F. H. Cano, J. Elguero, A. Baldy, M. Pierrot, M. M. T. Khurshid, J. B. Larcombe-McDouall, J. Am. Chem. Soc. 1989, 111, 7304-12) as well as solid state NMR (O. Klein, F. Aguilar-Parrilla, J. M. Lopez, N. Jagerovic, J. Elguero,|H.-H. Limbach, J. Am. Chem. Soc. 2004, 126, 11718) that $\mathrm{H}-32$ and $\mathrm{H}$-33 in simple pyrazole derivatives hop between the pyrazole nitrogens as a result of tautomerization. Using "water gate" or "WET" pulse sequences could allow seeing exchangeable protons.

Response: We did use "Watergate" for our recording of both $1 \mathrm{D}{ }^{1} \mathrm{H}$ and NOESY spectra. In our case, we see no pyrazole NH signals in the monomer at $298 \mathrm{~K}$. At $275 \mathrm{~K}$, on the contrary, we see new pyrazole $\mathrm{NH}$ signals appear for the hexamer. For the monomer, only doublets at positions 4, 18, 27, and 30 are observed at this temperature. We can thus deduce that the pyrazole tautomeric exchange operates even at $275 \mathrm{~K}$, but that the formation of the hexameric aggregate almost entirely prevents it and thus renders the pyrazole $\mathrm{NH}$ protons observable. The reason for this is most likely found in a unique very strong fourfold hydrogen bond assembly in the hexamer as depicted in Figure 2a.

Destabilization of 1 in water by THF addition: The original sample of monomer 1 in water had a concentration of $45 \mathrm{mM}$. At $275 \mathrm{~K}$, the integral of proton signals reveals $40 \%$ of the peptide to be in the hexameric form. Upon diluting to $23 \mathrm{mM}$, the ratio decreased to $15 \%$. Addition of $5 \%(\mathrm{~V})$ of THF almost completely dissolved the assembly. The ${ }^{1} \mathrm{H}$ spectra of the diluted sample and the one added with THF at $275 \mathrm{~K}$ are enclosed in Figures S12 and S13, respectively: 


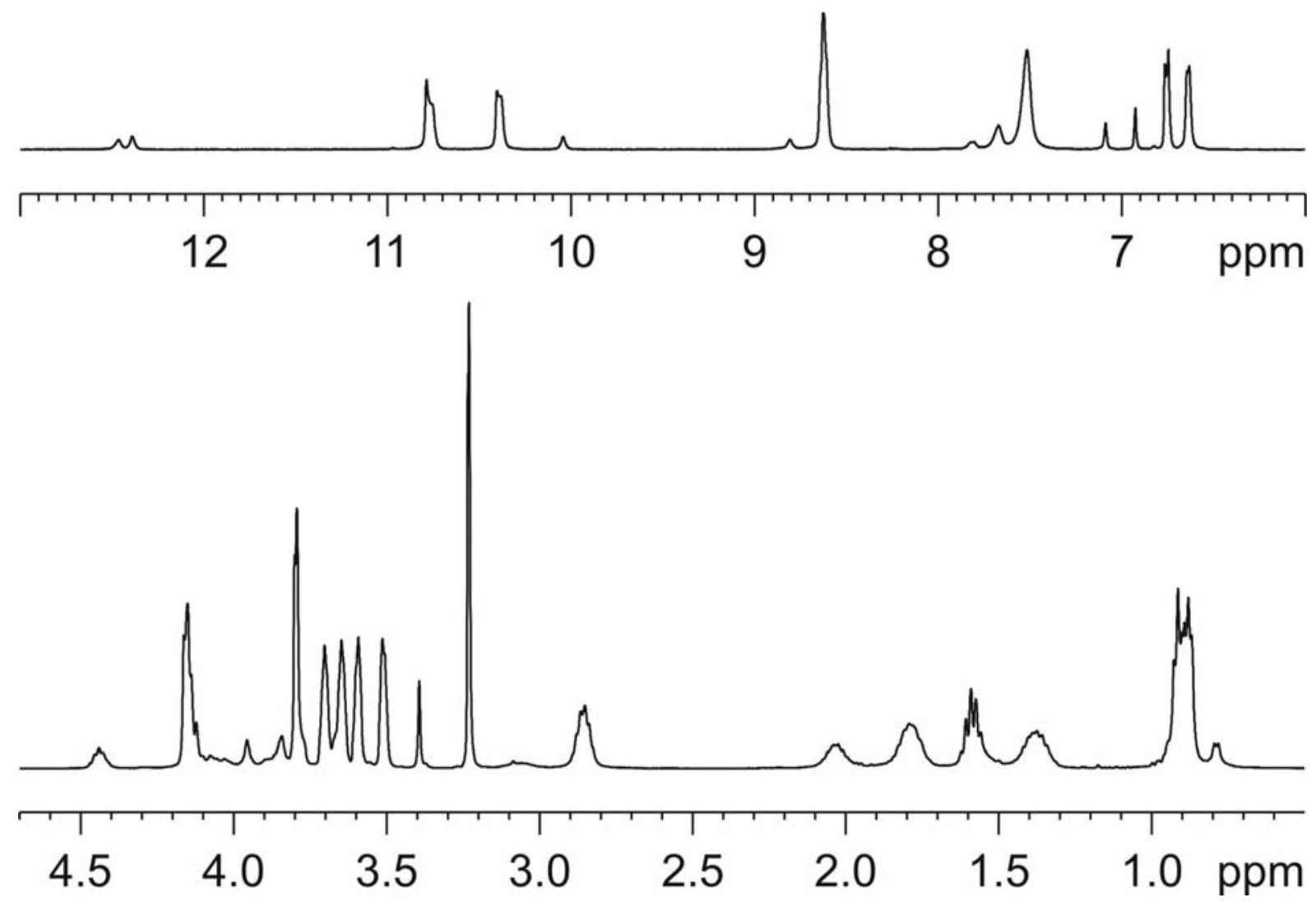

Figure S12: ${ }^{1} \mathrm{H}$ NMR spectrum of diluted 1 in water (23 mM): only $15 \%$ hexamer.
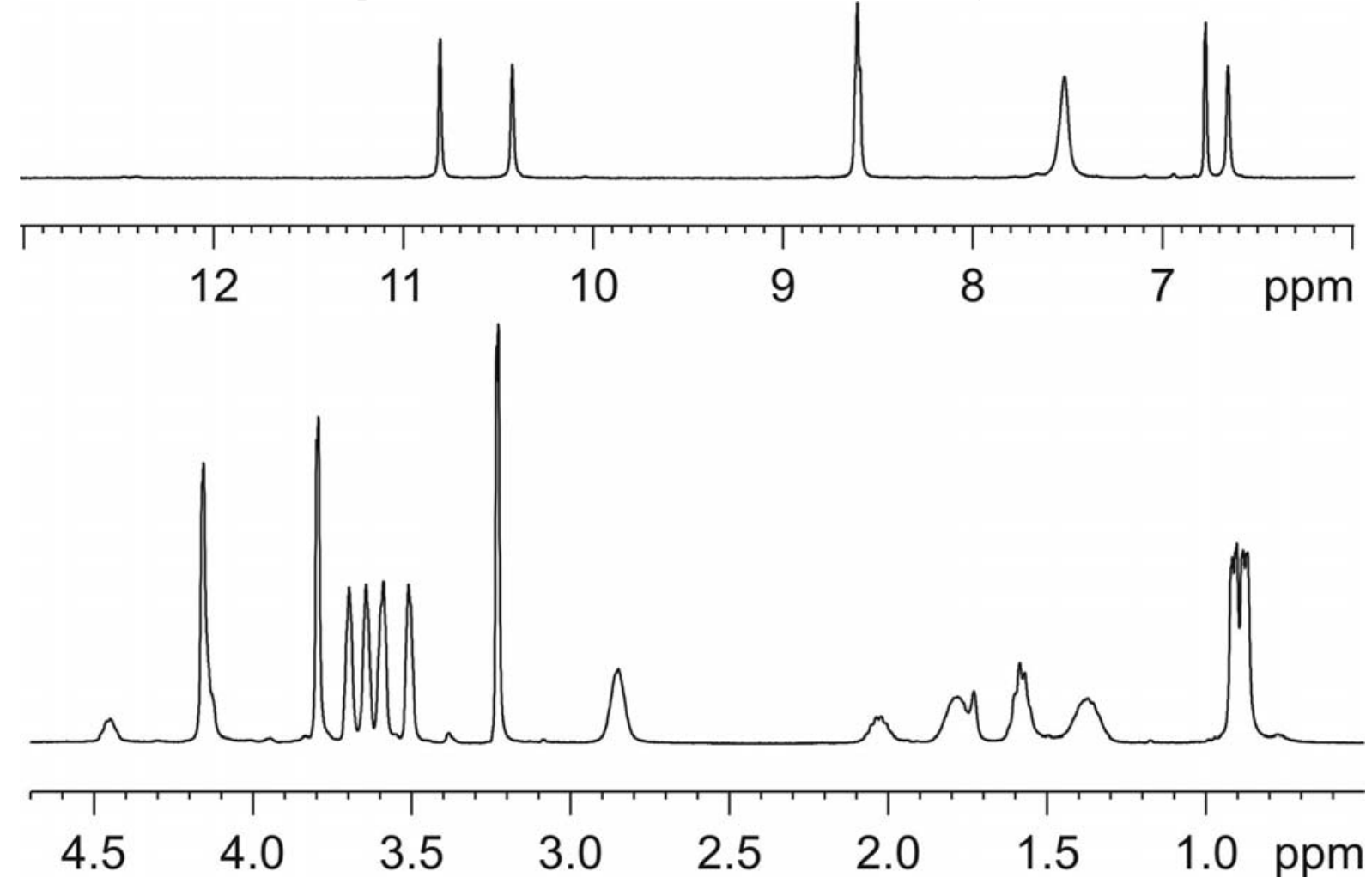

Figure S13: ${ }^{1} \mathrm{H}$ NMR spectrum of diluted 1 in water $(22 \mathrm{mM})$ with $5 \%$ added THF: almost no hexamer. 

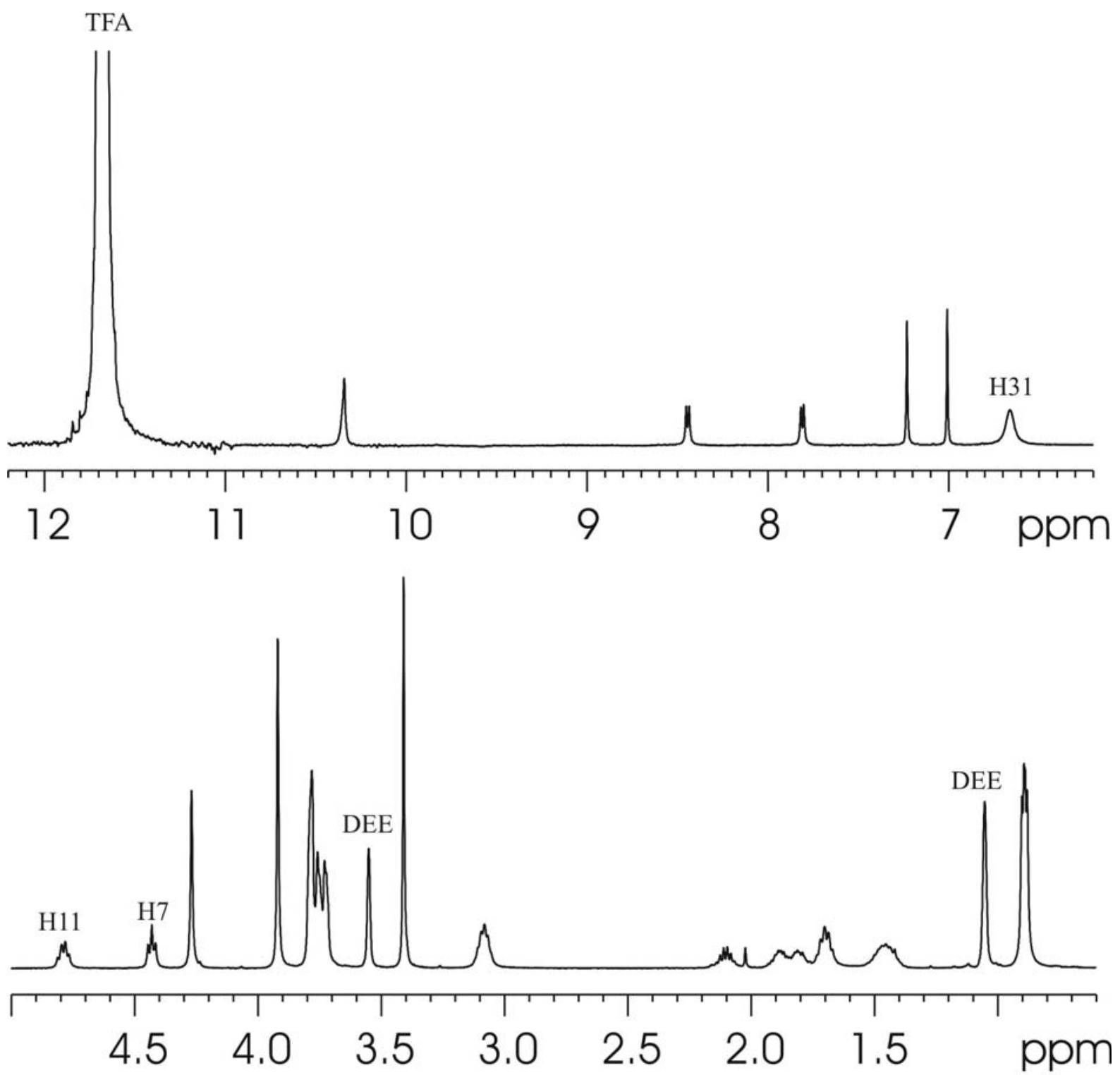

Figure S14: ${ }^{1} \mathrm{H}$ NMR spectrum of PzKVPz (1) in TFA/TFA-d $\mathrm{d}_{1}(9: 1)$ at $22 \mathrm{mM}$ with 30 Vol.$\%$ diethyl ether DEE- $\mathrm{d}_{10}$ at $298 \mathrm{~K}$. The addition of DEE did not bring any change to the spectrum of 1. 
Diffusion measurements of 1 in $\mathrm{H}_{2} \mathrm{O} / \mathrm{D}_{2} \mathrm{O}(9: 1)$ at $279 \mathrm{~K}$

\section{Experimental values:}

$\mathrm{D}_{\mathrm{H} 2 \mathrm{O}}=1.34 \times 10^{-9} \mathrm{~m}^{2} / \mathrm{s}$

$\mathrm{D}_{\text {monomer }}=1.59 \times 10^{-10} \mathrm{~m}^{2} / \mathrm{s} ; \mathrm{R}_{\mathrm{h}}=8.4 \AA$

$\mathrm{D}_{\text {hexamer }}=0.85 \times 10^{-10} \mathrm{~m}^{2} / \mathrm{s} ; \mathrm{R}_{\mathrm{h}}=15.6 \AA$

\section{Calculated values:}

Estimation of volume and radius of monomer, dimer, trimer, and hexamer within the Sybyl program:

\begin{tabular}{|l|l|l|l|}
\hline Oligomer & Approximated Shape & Volume $\left(\AA^{3}\right)$ & Spherical radius $(\AA)^{\mathrm{a}}$ \\
\hline Monomer & $/$ & $/$ & $/$ \\
\hline Dimer & $\begin{array}{l}\text { Cubic } \\
\mathrm{A}=4.0 \AA \\
\mathrm{B}=\mathrm{C}=9.0 \AA\end{array}$ & 324 & 4.2 \\
\hline Trimer & $\begin{array}{l}\text { Cylinder } \\
\mathrm{R}=10 \AA \\
\mathrm{H}=10 \AA\end{array}$ & 3140 & \\
& $\begin{array}{l}\text { Cylinder } \\
\mathrm{R}=10 \AA \\
\mathrm{H}=10 \AA\end{array}$ & & 8.8 \\
& \multicolumn{2}{|l|}{6280} & $\mathbf{1 1 . 4}$ \\
& \multicolumn{2}{|l}{} \\
\hline
\end{tabular}

a The spherical radius is defined as $R=\left(\frac{3 V}{4 \pi}\right)^{\frac{1}{3}}$.

Remarks:

1. The $\mathrm{R}_{\mathrm{h}}$ calculated from diffusion measurements includes a solvent sphere (here $\mathrm{H}_{2} \mathrm{O}$ ).

2. The $\mathrm{R}_{\mathrm{h}}$ was calculated with the assumption of a spherical shape of the molecule. Nonspherical particles move slower than their corresponding spherical analogs of the same volume. Thus the calculated $\mathrm{R}_{\mathrm{h}}$ here may overestimate the real molecular size to some extent.

3. The estimation of volume and radius of nonspherical molecules within any modelling program is very rough.

4. Assuming a cylindric model for the hexamer, a shape factor of 1.29 is obtained for the solvation shell. If this correction factor is applied to the experimentally determined $\mathrm{R}_{\mathrm{h}}$ value, the effective hydrodynamic radius for the hexamer becomes $12.1 \AA$. This value correlates well with the calculated value shown above in the table (11.4 $\AA$ ). For an explanation of the shape factor see X. Xie et al. (J. Am. Chem. Soc. 2003, 125, 1595-1601).
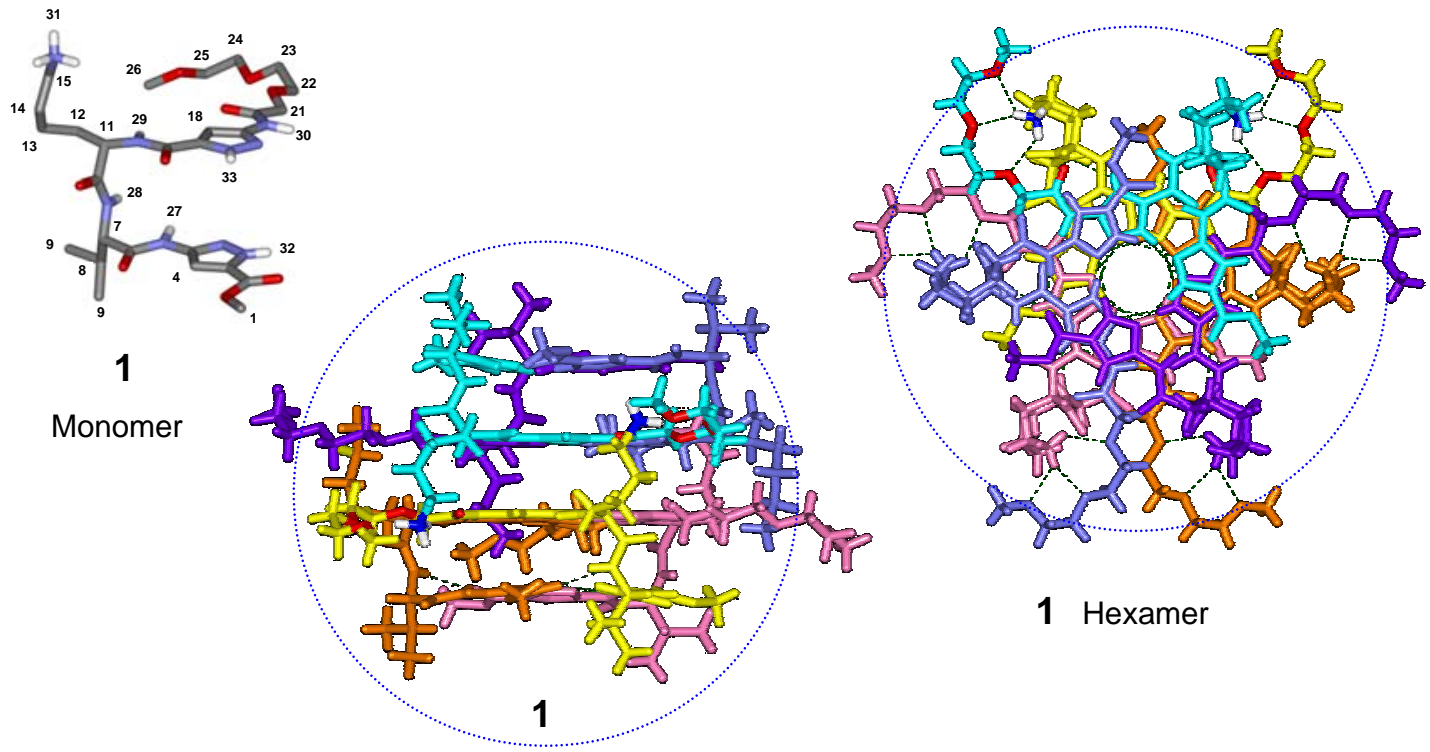

1 Hexamer

Hexamer 


\section{Quantum chemical calculations:}

\begin{tabular}{|c|c|c|c|}
\hline Proton & $\delta_{\text {calc }}[\mathrm{ppm}]$ & $\delta_{\exp }[\mathrm{ppm}]$ & $\delta_{\exp }-\delta_{\text {calc }}[\mathrm{ppm}]$ \\
\hline 1 & 4.0 & 3.8 & -0.2 \\
\hline 7 & 4.3 & 4.2 & -0.1 \\
\hline 8 & 1.4 & 2.0 & 0.6 \\
\hline 9 & 1.2 & 0.9 & -0.3 \\
\hline 11 & 4.2 & 4.4 & 0.2 \\
\hline 12 & 1.7 & 1.8 & 0.1 \\
\hline 13 & 2.1 & 1.4 & -0.7 \\
\hline 14 & 2.0 & 1.6 & -0.4 \\
\hline 15 & 3.4 & 2.9 & -0.5 \\
\hline 21 & 3.6 & 4.2 & 0.6 \\
\hline 22 & 3.3 & 3.7 & 0.4 \\
\hline 23 & 3.3 & 3.7 & 0.4 \\
\hline 24 & 3.2 & 3.6 & 0.4 \\
\hline 25 & 3.2 & 3.5 & 0.3 \\
\hline 26 & 3.2 & $3.2,3.3$ & 0.1 \\
\hline 4 & 7.9 & $6.7,6.8$ & -1.2 \\
\hline 18 & 7.5 & 6.6 & -0.9 \\
\hline 27 & 6.6 & 10.7 & 4.1 \\
\hline 28 & 5.5 & 8.6 & 3.1 \\
\hline 29 & 5.5 & 8.6 & 3.1 \\
\hline 30 & 6.9 & $10.3,10.4$ & 3.5 \\
\hline $31_{a / b / c}$ & $4.9 / 4.6 / 4.5$ & 7.6 & $2.7 / 3.0 / 3.1$ \\
\hline 32 & 9.8 & - & - \\
\hline 33 & 10.3 & - & - \\
\hline
\end{tabular}

\begin{tabular}{|c|c|c|c|}
\hline Proton & $\delta_{\text {calc }}[\mathrm{ppm}]$ & $\delta_{\exp }[\mathrm{ppm}]$ & $\delta_{\text {exp }}-\delta_{\text {calc }}[\mathrm{ppm}]$ \\
\hline 1 & 4.2 & 4.0 & -0.2 \\
\hline 7 & 4.7 & - & - \\
\hline 8 & 1.6 & 2.0 & 0.4 \\
\hline 9 & 1.2 & $0.8,1.0$ & -0.3 \\
\hline 11 & 4.3 & - & - \\
\hline 12 & 1.5 & $1.8 ?$ & 0.3 \\
\hline 13 & 2.0 & - & - \\
\hline 14 & 2.4 & 1.6 & -0.8 \\
\hline 15 & 3.0 & 3.1 & 0.1 \\
\hline 21 & 3.8 & $3.9,4.1$ & 0.2 \\
\hline 22 & 4.0 & - & - \\
\hline 23 & 3.3 & 4.1 & 0.8 \\
\hline 24 & 3.5 & - & - \\
\hline 25 & 3.5 & - & - \\
\hline 26 & 3.5 & 3.4 & -0.1 \\
\hline 4 & 7.6 & 7.0 & -0.6 \\
\hline 18 & 6.2 & 7.1 & 0.9 \\
\hline 27 & 9.5 & 10.1 & 0.6 \\
\hline 28 & 6.6 & 8.8 & 2.2 \\
\hline 29 & 5.1 & 8.8 & 3.7 \\
\hline 30 & 11.2 & 10.8 & -0.4 \\
\hline $31_{a^{\prime} / b^{\prime} / c^{\prime}}$ & $8.4 / 7.4 / 3.5$ & 7.7 & $-0.7 / 0.3 / 4.2$ \\
\hline 32 & 11.9 & 12.5 & 0.6 \\
\hline 33 & 13.4 & 12.4 & -1.0 \\
\hline
\end{tabular}

Table S8. Quantum chemical shift calculations for monomer and hexamer.

Left: ${ }^{1} \mathrm{H}$ NMR relative chemical shifts for the monomer (HF/6-31G**) for C-Hs (upper part of table) and N-Hs (lower part). For numbering see Fig. S1. Experimental shifts were measured in $\mathrm{H}_{2} \mathrm{O}: \mathrm{D}_{2} \mathrm{O}$ (9:1) at $275 \mathrm{~K}$. The structure was optimized by force-field (MMFF94/ $\left.\mathrm{H}_{2} \mathrm{O}\right)$ without constraints.

Right: ${ }^{1} \mathrm{H}$ NMR chemical shifts for the hexamer (HF/6-31G**) for C-Hs (upper part of table) and NHs (lower part). Experimental shifts were measured in $\mathrm{H}_{2} \mathrm{O}: \mathrm{D}_{2} \mathrm{O}(9: 1)$ at $275 \mathrm{~K}$. The structure is an Xray structure where the positions of the hydrogens were optimized by force-field (MMFF94/ $\mathrm{H}_{2} \mathrm{O}$ ).

In order to support the hypothesis of the hexamer-formation in aqueous solution, quantum chemical ${ }^{1} \mathrm{H}$ NMR shift calculations have been performed at the GIAO-HF/6-31G** level for the monomeric and hexameric structures of the peptide, respectively. As a starting point for the calculations, the hexamer X-ray structure was employed for which the proton positions were optimized at the force-field level (Macromodel 9.1, MMFF94/ $\mathrm{H}_{2} \mathrm{O}$ ). The structure of the monomer was computed by using one fragment of the X-ray structure of the hexamer as a starting point for the optimization with the force-field (Macromodel 9.1, MMFF94/H2O) without imposing any constraints.

While it has to be stressed that no solvent effects have been included in the chemical-shift calculations, we expect that those protons which are not potentially involved in hydrogen bonds with the solvent and in particular the protons forming the intermolecular bonds between the monomer units in the hexamer should be well described. This can indeed be seen from the data listed in Table S8 : The computed chemical shifts of the protons 27, 30, 32, and 
33 involved in the intermolecular hydrogen bonds of the hexamer containing a total of 534 atoms (5370 basis functions) agree within $0.6 \mathrm{ppm}$ (protons no. 27, 30, 32) and $1.0 \mathrm{ppm}$ (no. 33) with the experimental data, which supports the hypothesis of a hexamer formation. The larger deviation for proton 33 might be due to solvent effects. The experimental NMR signals for the monomer and the hexamer (see Fig. S10) are quite similar, so that a distinction by means of an assignment using the calculated NMR data is difficult. Nevertheless, the experimental and theoretical NMR data for both the monomer and the hexamer are compared in the following.

The calculated ${ }^{1} \mathrm{H}$ NMR shifts for the monomer are presented in the left part of Table S8, as well as the monomer signals measured experimentally at $275 \mathrm{~K}$ (marked light blue in Fig. S10). Table S8 is composed of two parts, where the upper part lists all protons connected to carbon atoms and the lower part protons connected to nitrogen atoms, respectively. For the protons connected to carbon all differences between experiment and theory are within 0.7 ppm and therefore within the error bars, with the exception of protons no. 4 and 18, for which the difference is slightly larger with 1.2 and $0.9 \mathrm{ppm}$, respectively. These latter protons, however, show a stronger solution dependence of the NMR chemical shifts than the other protons ( 0.3 to $0.4 \mathrm{ppm}$ when comparing the experimental values in $\mathrm{H}_{2} \mathrm{O}$ and DMSO) which may explain the larger difference between experiment and theory, since all calculations were performed without solvent. The protons connected to nitrogen (lower part of table) are calculated to be too strongly shielded when comparing to the experiment. The deviations are in the order of 3 to $4 \mathrm{ppm}$ and can be rationalized by hydrogen bonds with these protons in polar solutions like $\mathrm{H}_{2} \mathrm{O}$ and DMSO, which are not accounted for in the calculations.

The right part of Table S8 shows the calculated ${ }^{1} \mathrm{H}$ NMR shifts for the hexamer unit, together with the hexamer signals measured at $275 \mathrm{~K}$ (marked red in Fig. S10). Here, all calculated chemical shifts were averaged over the six monomers of the complex, so that the numbering is analogous to the monomer case. Chemical shift differences between the individual monomers of the hexamer can considered to be negligible ( $0.1 \mathrm{ppm}$ at maximum). While the chemical shifts for protons involved in the hydrogen bridges forming the hexamer agree nicely with the experimental data and have already been discussed above, the remaining protons are discussed in the following: The calculated chemical shifts for all protons bound to carbon agree with the experimental values within 0.9 ppm. In contrast, protons no. 28 and 29, which do not take part in hydrogen bonds between monomer units and are expected to interact strongly with solvent molecules cannot be described by the chosen gas phase model. A special case is proton no. 31 (which marks the average over the ammonium protons): here, two protons are involved in hydrogen bridges forming the hexamer complex and agree within 0.3 and $0.7 \mathrm{ppm}$ with the experimental average value, respectively, while only the third proton is expected to form hydrogen bridges to the solvent, so that it cannot be described by our present model (which explains the deviation of $4.2 \mathrm{ppm}$ for this proton as compared to the experimental average value).

While most of the calculated shifts for the monomeric and hexameric unit could be assigned to both of the two sets of experimental signals (apart from those protons strongly influenced by solvent effects missing in our model), the hexamer formation is most strongly supported by the good agreement between calculated and experimental ${ }^{1} \mathrm{H}$ NMR shifts for protons no. $27,30,32,33$ and two of the ammonium protons (average value; proton no. 31). These protons are involved in intermolecular hydrogen bonding between the monomer units forming the hexamer structure. 


\section{Force-field calculations}

Force-field calculations were carried out with the program MacroModel 9.0, using the MMFF force-field and water as a GBA continuum model.

The crystal structure was refined by molecular mechanics calculations, resulting in slightly shifted hydrogen positions for optimized hydrogen bonding. Subsequent Monte-Carlo simulations were conducted without any constraints for 1000 steps in water and chloroform. In general, geometrical differences within the new unique stored structures (compared to the crystal structure) were found to be much smaller in chloroform than in water. In the latter solvent, the best unique structure was found to be $\sim 80 \mathrm{~kJ}$ less stable than the original starting structure (Fig. S15). In all consecutive (even less stable) structures the half-crown locks were opened and the arms twisted into the solvent space. In addition, distortions of the pyrazole nuclei and the triplexes were found, although the overall hydrogen bond pattern as well as the stacking were maintained. Finally, a Molecular dynamics run was performed for a whole nanosecond (1000 ps) which invariably led to energetic movements of the half-crown arms as well as the stacked planes, but throughout the entire time period the overall hydrogen bond pattern and the four stacked planes were retained. It should be kept in mind, that in force-field calculations stacking is only calculated as (enthalpic) van-der-Waals attraction, since no entropic contribution can be implemented, as it is operating in the classical hydrophobic effect. Thus we conclude from these rough estimates, that the core unit is very stable, especially due to dispersive forces.
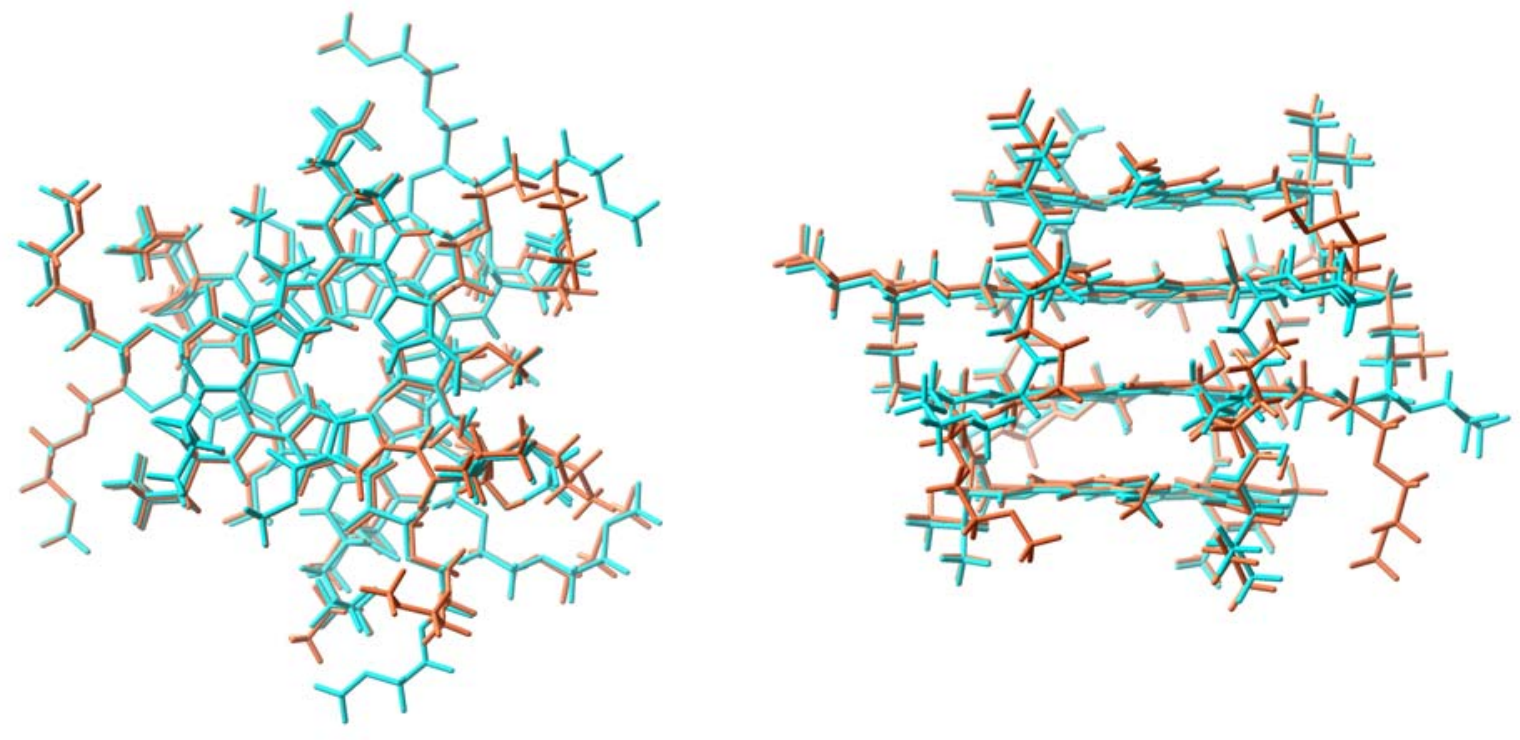

Figure S15: Monte Carlo simulation of the hexameric aggregate 1 (MacroModel 9.0, water, 1000 steps): best unique structure (brown) next to the original starting structure (blue, relative energy: + $75 \mathrm{~kJ} / \mathrm{mol}$ ). 